\title{
Vasculitides primarily involving the skin
}

\author{
Ph.D. Thesis
}

GÉZA RÓBERT NAGY M.D.

Supervisor: Zsuzsanna Bata-Csörgő M.D., Ph.D., D.Sc.

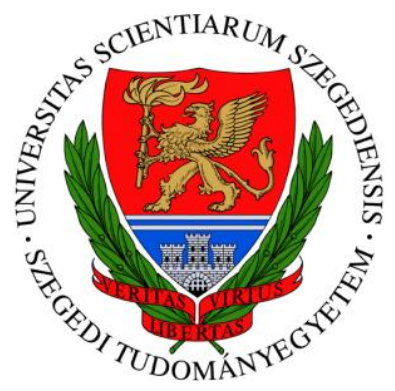

Department of Dermatology and Allergology

Faculty of Medicine

University of Szeged

Szeged

2020 . 


\section{TABLE OF CONTENTS}

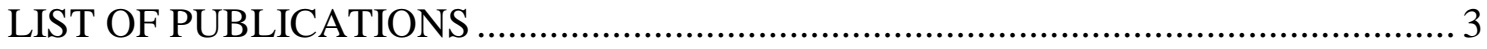

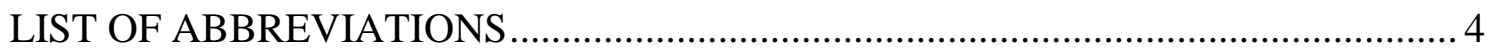

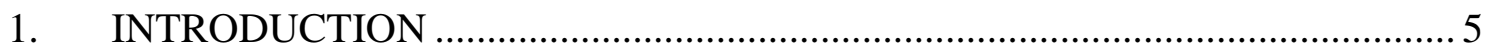

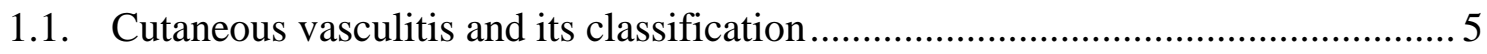

1.2. Immunoglobulin A vasculitis and its systemic involvement ............................ 8

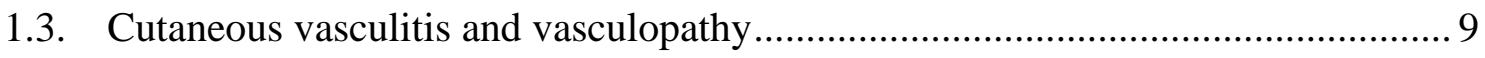

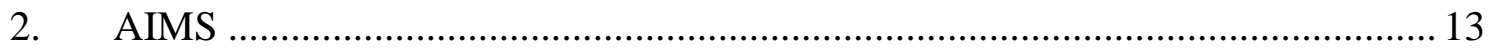

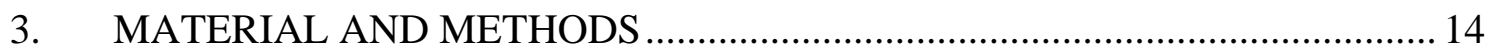

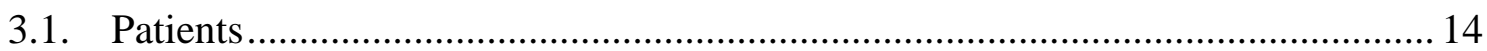

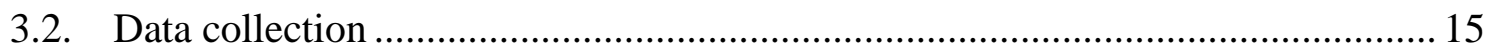

3.3. Assessment of GI and renal involvement in IgAV ...................................... 15

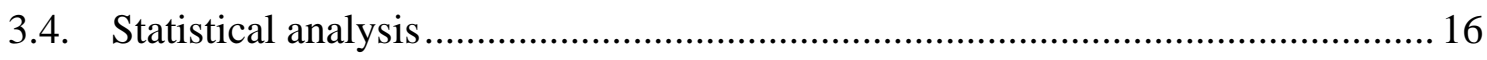

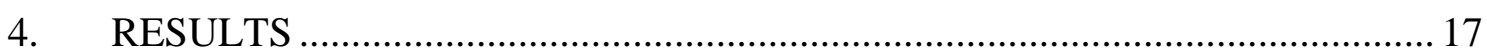

4.1. Predictors of systemic involvement in adult IgAV patients (Study I.) ................ 17

4.1.1. Basic characteristics of the study sample .................................................... 17

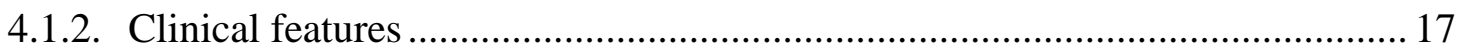

4.1.3. Receiver operating characteristic curves of NLR versus other inflammatory

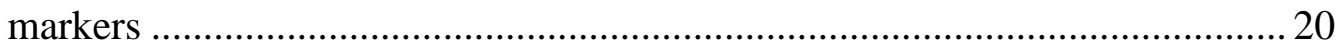

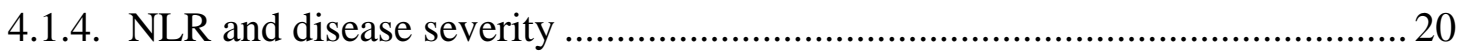

4.1.5. Assessing the applicability of NLR as a prognostic indicator in clinical practice 22

4.2. Assessing the presence of vasculopathy in adult CPAN patients (Study II.) ....... 23

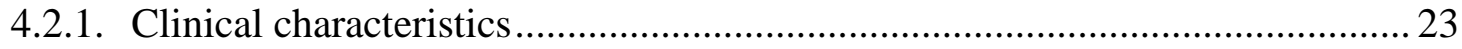

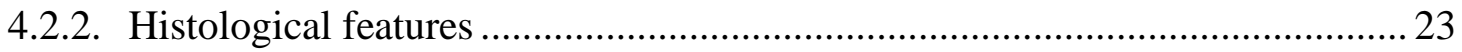

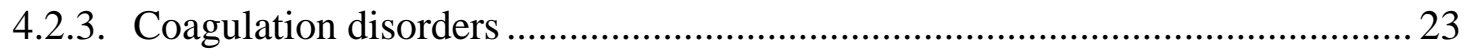

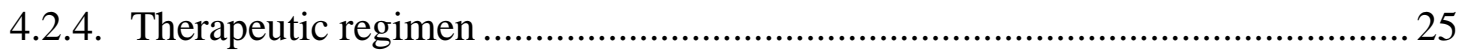

4.3. Difficulty in the segregation of vasculitis and vasculopathy in clinical practice 26

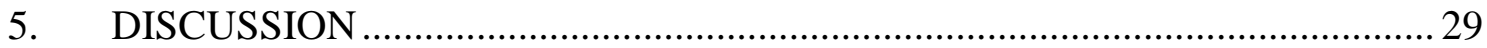

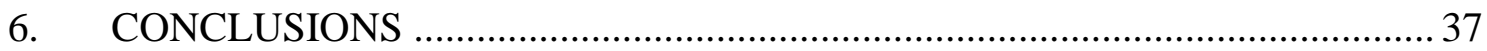

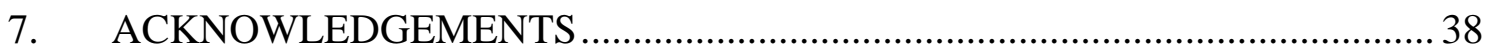

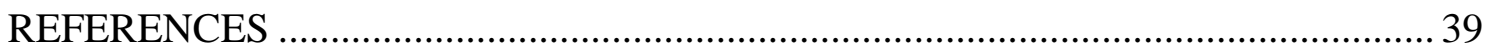




\section{LIST OF PUBLICATIONS}

\section{This doctoral thesis is based on the following publications:}

I. Nagy GR, Kemény L, Bata-Csörgő Zs. Neutrophil-to-lymphocyte ratio: a biomarker for predicting systemic involvement in adult IgA vasculitis patients. J Eur Acad Dermatol Venereol. 2017; 31(6): 1033-1037. IF: 4.287

II. Nagy GR, Veres K, Belső N, Németh IB, Varga E, Korom I, Kemény L, Bata-Csörgő Zs. Focal vascular occlusion: a link between livedoid vasculopathy and cutaneous polyarteritis nodosa? Manuscript under review at J Am Acad Dermatol Int.

III. Nagy GR, Kovács L, Németh IB, Varga E, Kemény L, Bata-Csörgő Zs. AntiInterleukin-6 receptor therapy-induced cutaneous symptoms resembling purpura fulminans in a patient with seropositive rheumatoid arthritis. J Eur Acad Dermatol Venereol. 2020 doi: 10.1111/jdv.16442 [Epub ahead of print]

Total impact factor of original papers directly related to the thesis: 4.287

\section{Publications not directly related to the thesis:}

IV. Tóth EJ, Nagy GR, Homa M, Ábrók M, Kiss IÉ, Nagy G, Bata-Csörgő Zs, Kemény L, Urbán E, Vágvölgyi C, Papp T. Recurrent Scedosporium apiospermum mycetoma successfully treated by surgical excision and terbinafine treatment: a case report and review of the literature. Ann Clin Microbiol Antimicrob. 2017; 16(1): 31. IF: 3.155

V. Belső N, Nagy GR, Korom I, Varga E, Németh I, Szolnoky Gy, Kemény L, Bata Zs. A bőrtünetek jelentősége az EGPA (eosinophil granulomatosis polyangiitissel) diagnosztikájában [The significance of cutaneous manifestations in EGPA (eosinophilic granulomatosis with polyangiitis)]. Hungarian Journal of Dermatology and Venerology. 2019; 95(2): 48-52.

Total impact factor of original papers not related to the thesis: 3.155 


\section{LIST OF ABBREVIATIONS}

$\begin{array}{ll}\text { ANCA } & \text { antineutrophil cytoplasmic antibody } \\ \text { CHCC } & \text { Chapel Hill Consensus Conference } \\ \text { CPAN } & \text { cutaneous polyarteritis nodosa } \\ \text { CRP } & \text { c-reactive protein } \\ \text { CYP } & \text { cytochrome P450 } \\ \text { ESR } & \text { erythrocyte sedimentation rate } \\ \text { GI } & \text { gastrointestinal } \\ \text { IgAV } & \text { immunoglobulin A vasculitis } \\ \text { IL-6 } & \text { interleukin-6 } \\ \text { INR } & \text { international } \\ \text { IQR } & \text { interquartile range } \\ \text { LV } & \text { livedoid vasculopathy } \\ \text { MCV } & \text { anti-mutant-citrullinated vimentin } \\ \text { MPA } & \text { microscopic polyangiitis } \\ \text { MPO } & \text { myeloperoxidase } \\ \text { NETs } & \text { neutrophil extracellular traps } \\ \text { NLR } & \text { neutrophil-to-lymphocyte ratio } \\ \text { PAN } & \text { polyarteritis nodosa } \\ \text { PAN } & \text { polyarteritis nodosa } \\ \text { PR3 } & \text { proteinase } 3 \\ \text { RA } & \text { rheumatoid arthritis } \\ \text { ROC } & \text { receiver operating characteristic curve } \\ \text { TD } & \text { standard deviation } \\ \text { TCE } & \text { tocilizumab } \\ \text { Thite blood cell }\end{array}$




\section{INTRODUCTION}

\subsection{Cutaneous vasculitis and its classification}

The term vasculitis denotes inflammation of the blood vessel wall, which can occur in any organ system of the human body. In terms of blood vessel involvement, a stratification can be made in accordance whether the vasculitis affects the small, medium and the largesized vessels of the arterial and/or the venous systems. Notably, certain vasculitides may have multiple vessel-type and/or size involvements resulting in a heterogenous clinical manifestation, often making the diagnostic process difficult. The very first International Chapel Hill Consensus Conference Nomenclature of Vasculitides (CHCC) was conducted in 1994. Since then, several advances have been made with regards to understanding the pathophysiological processes behind each vasculitis, which is reflected by the addendum to the CHCC held in 2012, where the discussion of skin-limited or skin-dominant forms was also necessitated (Table 1) (1). Additionally, the CHCC work group's definition of skin also includes the subcutaneous layer (therefore incorporating the medium vessels as well) and the mucosa, further widening the disease spectrum.

Vasculitis of the skin (cutaneous vasculitis) may be categorized into three different disease forms: 1) skin component of a systemic vasculitis (e.g., skin symptoms of a systemic IgA vasculitis); 2) skin-limited form of a systemic vasculitis (e.g., skin-limited IgA vasculitis); and 3) single-organ vasculitis of the skin (e.g., cutaneous polyarteritis nodosa). While the latter does not evolve into a systemic form of vasculitis, skin-dominant variants may do so. It should be noted however, that nonspecific symptoms of systemic inflammatory processes, such as elevated inflammatory markers, leukocytosis or arthralgia do not necessarily establish sufficient indication for the presence of a systemic involvement of said vasculitis. Hence, proper exclusion of the presence of clinically detectable non-cutaneous organ involvement is required to conclude whether the patient truly has a skin-limited vasculitis or a single-organ vasculitis of the skin.

Cutaneous vasculitides are characterised by inflammation of either the small vessels (including arterioles, capillaries and postcapillary venules) in the superficial and mid dermis and/or the medium-sized vessels (including smaller arteries and veins) located in the deep dermis or subcutaneous fat tissue. This locality of inflammation thus determines both the clinical and histopathological presentation of said vasculitides. Additional features, such as the presence or absence of systemic involvement, the quality of the immunoglobulin 
Skin involvement status

CHCC2012

vasculitis category, name
Cutaneous component of systemic vasculitis
Skin-limited or skin-dominant variant

\section{Large vessel vasculitis}

Takayasu arteritis

$\begin{array}{ll}\text { No } & \text { No } \\ \text { Rare } & \text { No }\end{array}$

Giant cell arteritis

Rare

No

\section{Medium vessel vasculitis}

Polyarteritis nodosa

Yes

Yes

Kawasaki disease

No

No

\section{Small vessel vasculitis}

Microscopic polyangiitis

Granulomatosis with polyangiitis

Eosinophilic granulomatosis with polyangiitis

Anti-glomerular basement membrane disease

IgA vasculitis (Henoch-Schönlein)

Hypocomplementemic urticarial vasculitis (anti-C1q vasculitis)

$\begin{array}{cc}\text { Yes } & \text { Yes } \\ \text { Yes } & \text { Yes } \\ \text { Yes } & \text { Yes } \\ \text { No } & \text { No } \\ \text { Yes } & \text { Yes } \\ \text { Yes } & \text { Yes }\end{array}$

\section{Variable vessel vasculitis}

Behcet's disease

Cogan's syndrome

$$
\text { Yes }
$$$$
\text { Yes }
$$

Rare

No

\section{Vasculitis associated with systemic disease}

SLE, rheumatoid arthritis, sarcoidosis, etc.

\section{Vasculitis associated with probable etiology}

Drugs, infections, sepsis, autoimmune diseases, etc.

\section{Cutaneous SOV (not included in CHCC2012)}

$\operatorname{IgM/IgG~vasculitis~}$

Nodular vasculitis (erythema induratum of Bazin)

Erythema elevatum et diutinum

Hypergammaglobulinemic macular vasculitis

Normocomplementemic urticarial vasculitis
No (not observed yet) Yes (as SOV)

No

Yes (as SOV)

No

Yes (as SOV)

No

Yes (as SOV)

No

Yes (as SOV)

Table 1. Skin involvement status by vasculitis category and disease

CHCC2012, 2012 revised International Chapel Hill Consensus Conference Nomenclature of Vasculitides; SLE, systemic lupus erythematosus; SOV, single-organ vasculitis. 
deposition found with direct immunofluorescence staining and the presence of antineutrophil cytoplasmic antibodies add further criteria to be considered.

While multiple subclassifications are possible, the two major pathophysiological variants are antineutrophil cytoplasmic antibody (ANCA)-associated vasculitis and immune complex-mediated vasculitis. As the clinical manifestations of ANCA-associated vasculitides show great heterogeneity, they may further be categorized based on their unique epidemiologic, pathologic and clinical features, in addition to their immunoserological findings, such as the presence of anti-proteinase 3 (anti-PR3) or anti-myeloperoxidase antibodies (anti-MPO).

Immune complex vasculitides -as the name entails- are mediated by immune complexes, which first form in the presence of antigen and then later precipitate in antigen excess. Immune complex deposition primarily takes place in the dermal postcapillary venules, where inflammation is induced through the activation of the complement cascade, resulting in mast cell degranulation and neutrophil chemotaxis, thus it can also be histopathologically characterized as leukocytoclastic vasculitis. Cutaneous symptoms usually develop a week after antigen exposure, in the form of palpable purpuras, erythematous papules, vesicles or urticas. Additional symptoms may also include fever, myalgia, weight loss, arthralgia, arthritis or various internal organ involvements (e.g.: genitourinary, renal, gastrointestinal and neurologic involvement) (2). In systemic vasculitides, the signs of organ involvement often precede the development of the cutaneous symptoms, ranging from as short as a few days to as long as years (3). Proper skin biopsy sampling of the inflamed vessels increases the diagnostic yield, where the ideal timespan of the fresh cutaneous lesions is within the first 24 to 48 hours of its appearance. In the majority of cases a punch biopsy may suffice, however an incisional biopsy may be preferred, if the clinical manifestations suggest the involvement of the deeper, medium-sized vessels, such as in the appearance of necrosis or ulceration. The classic, initial histologic findings show leukocytoclastic vasculitis with the infiltration of the vessel walls (predominantly in the postcapillary venules) by neutrophils, leukocytoclasia (fragmentation of neutrophils, resulting in nuclear dust) and extravasation of erythrocytes. As the inflammation progresses, the histopathologic feature is later characterized by mononuclear infiltrate. Direct immunofluorescent staining of the cutaneous biopsy specimen shows deposition of either $\mathrm{C} 3, \operatorname{IgA}, \operatorname{IgG}$ and/or IgM in a granular pattern within the vessel walls. The highest chance of detecting immunoglobulin deposition is performing a biopsy from a lesion that is present 
withing the first 48 hours. Of note, the evolution of the vasculitis may alter the qualitative features of direct immunofluorescent findings and can also result in its negativity (3). Hence, biopsy from a fresh lesion is essential for proper diagnostic workup. A key example for this was within the 2012 revised CHCC, where the denomination "Henoch-Schönlein purpura" was replaced by the term "IgA vasculitis", owing to the compelling data indicating that IgA deposition proposes a unique pathophysiological feature of this vasculitis, segregating it from other immune complex-mediated forms.

\subsection{Immunoglobulin A vasculitis and its systemic involvement}

Immunoglobulin A vasculitis (IgAV), formerly known as Henoch-Schönlein purpura, is an immune complex-mediated small-vessel leukocytoclastic cutaneous vasculitis, characterized by palpable purpura, accompanied by arthralgia or arthritis, gastrointestinal (GI) and renal involvement (also known as the "classic triad" of IgAV) (4). While the exact cause of the disease is unknown, it is evident that the $\operatorname{IgA}$ system has a key role in its pathophysiology, as serum IgA1 level elevation have been demonstrated in patients, owing to the increase in their production, disturbance in their clearance and their aberrant glycosylation $(5,6)$. In most cases, the symptoms are usually benign and self-limiting in the acute stage, which can even resolve spontaneously, without a therapeutic intervention. However, in a small percentage of patients, severe systemic involvement may develop and life-threatening complications may occur, requiring aggressive treatment modalities. Cutaneous symptoms in the form of palpable purpura is nearly always apparent in $\operatorname{IgAV}$ patients, which are more commonly localized to the pressure areas, particularly around the ankle regions. In terms of joint involvement, arthralgia most frequently affects the knees and the ankles, likely due to the high pressure in the venule complexes associated with the production of the synovial fluid, thus allowing for a favorable flow kinetic for the immune complex deposition. GI involvement may precede the onset of purpuras, presenting with colicky abdominal pain and/or GI bleeding. These symptoms are cause by bowel ischemia and oedema, which may further proceed into infarction or perforation. Renal involvement occurs in approximately $40-50 \%$ of patients, presenting with microscopic hematuria, which may be accompanied by proteinuria. Of note, nephritis may have a prolonged development timespan, compared to the cutaneous lesions, and can also be clinically detectable within 36 months as well. The distinction between IgAV with nephritis and IgA nephropathy should be made, despite their clinical similarities. Both entities are characterized by hematuria, proteinuria and immune complex deposition in the glomerular mesangium. The main 
differentiator for the two is that while $\operatorname{Ig} \mathrm{A}$ nephropathy is restricted specifically to the kidneys, $\operatorname{Ig}$ A with nephritis is part of the clinical spectrum of a systemic involvement (7). $\mathrm{IgAV}$ is often regarded as a disease of childhood with an annual incidence rate of 3$26.7 / 100.000$, but contrary to popular belief, it is not uncommon in adults $(6,8)$. Although it is considered to be the same entity, the clinical manifestations and disease course differ greatly in these two age groups. Previous studies have demonstrated that unlike in children, adult patients develop systemic involvement more frequently, with a high risk of severe GI bleeding and chronic kidney disease $(9,10)$. This highlights the importance and the need of prognostic markers that can help identify IgAV patients who are at risk of developing unfavorable extracutaneous manifestations.

While predictive factors have been extensively studied in children, there is limited data on adults (11-13). Blood neutrophil-to-lymphocyte ratio (NLR) is an inexpensive and easily obtainable laboratory marker for quantifying systemic inflammation, which has been used to predict clinical outcomes in patients with various internal malignancies, cardiovascular disease and liver cirrhosis (14-18). NLR is defined by the absolute number of neutrophils divided by the absolute number of lymphocytes. Neutrophilia is often accompanied with lymphocytopenia, indicating a decreased cell-mediated adaptive immune response. As this ratio integrates information on two immune pathways, it may provide a predictive ability that outweighs other inflammatory parameters. Despite the strong prognostic potential of NLR in the aforementioned diseases, particularly in certain malignancies, no biopsy-proven IgAV studies have been conducted among the adult population. In this matter, if NLR harbors adequate prognostic potential in $\operatorname{IgAV}$, it may serve as not only a valuable biomarker in clinical decision making but a tool for patient risk stratification.

\subsection{Cutaneous vasculitis and vasculopathy}

In addition to the stratification of a "skin-limited" variant from a "systemic vasculitis with a skin component", another subject arises. In clinical practice, distinguishing vasculitis from vasculopathy is also a constant challenge, often leaving one contemplating whether thrombosis was secondary to the inflammation of the blood vessels or vice versa. Although current knowledge advocates segregation by means of histopathological examination, it may not grasp the whole underlying pathomechanisms, due to disease evolution, which may also explain the discrepancy seen in the literature regarding this question. Hence, one might consider the notion that owing to such underlying dynamic pathomechanisms, both vasculitis and vasculopathy may complementarily coincide with one another, and that diagnostic 
stratification based on histopathology alone may not grasp the full scope of the condition at hand. As such, rather than segregating thrombosis and inflammation, we should perhaps consider them as integrants of a framework, encompassing both vasculitis and vasculopathy; a phenomenon which has previously been observed in other entities $(19,20)$. Of peculiar interest, livedoid vasculopathy (LV) and cutaneous polyarteritis nodosa (CPAN) stand in the precipice of such a differential diagnostic challenge, where histopathology provides the distinction between the two entities.

Livedoid vasculopathy is an uncommon skin disease, characterized by focal vascular occlusions in the form of non-inflammatory thrombosis of the dermal venules, resulting in ulcerations and Milian's atrophie blanche (21). The denomination "atrophie blanche" has long been used as both a descriptive term for ivory-white scars on the lower limbs, thought to be a result of chronic inflammation and microvascular occlusion and also as a diagnostic term in the case of LV. Notably, LV was initially described as a clinical manifestation of vasculitis, however, at present time, the main pathogenic mechanism driving it is considered to be vaso-occlusive processes, primarily deriving from intraluminal thrombosis (22). This notion is supported by the vast body of literature describing the positive therapeutic effects of fibrinolytic, antiplatelet therapy and anticoagulants in LV. The main acute complication due to these focal non-inflammatory thromboses is pain, which is later followed by atrophic scarring, ulceration, mononeuritis multiplex, hyperpigmentation and cutaneous hemosiderosis (23). During the disease evolution, a chronic but benign clinical course can be seen with intermittent recurrences and exacerbations. Livedo racemosa can often be seen, generally localized to the lower extremities. The possible involvement of the peripheral nervous system is thought to be the result of ischemia deriving from the deposition of fibrin or the formation of thrombi in the vasa nervorum (24). A number of publications have advocated the concept of LV being a cutaneous manifestation of certain prothrombotic processes, rather than a unique pathology in itself. Therefore, a broad number of diseases have been associated with LV through the disturbance of the Virchow's triad, whether it be due to the disruption of the blood stasis in hyperviscosity syndromes, endothelial injury in systemic autoimmune diseases or hypercoagulability in thrombophilias (such as factor $\mathrm{V}$ Leiden mutation, protein $\mathrm{C}$ or S deficiency, elevated plasminogen activator inhibitor type 1) $(23,25)$. Due to the heterogenous etiology and wide differential diagnosis of LV, histopathological examination is essential. The histopathological features of LV are characterized by occlusion of the superficial and/or middle dermal vessels by fibrin 
deposition, intravascular thrombosis, segmental hyalinization and endothelial proliferation, all in the absence of vasculitis. Notably, such manifestations may also be apparent in the deep dermis. Deep biopsies are therefore necessary, as there are cases presenting with symptoms and histopathologic features suggestive of LV, but are actually the manifestations of CPAN (26).

Cutaneous polyarteritis nodosa is a skin-limited necrotizing vasculitis, affecting the small and medium sized arteries in the dermal-subcutaneous junction, resulting in the aforementioned "atrophie blanche" skin symptoms (1). Notably, the classification schemes of polyarteritis nodosa (PAN) and other vasculitides have changed as our knowledge of their aetiopathogenesis evolved over time, also allowing for more specific treatment modalities in these disorders. The term necrotizing vasculitis is now regarded as a feature of a wide variety of diseases with different etiologies, which include classic PAN, microscopic polyangiitis (MPA), granulomatosis with polyangiitis (formerly known as Wegener granulomatosis) and eosinophilic granulomatosis with polyangiitis (formerly known as Churg-Strauss syndrome). It should be mentioned that distinguishing PAN from MPA proposed a major challenge and as a result, MPA has often been mentioned as microscopic polyarteritis, indicating it to be on the same spectrum as PAN.

Subsequent studies have confirmed however, that MPA can be associated with ANCAs, thus establishing a distinction between the two. Furthermore, while PAN is not considered to be an inherited disease, certain mutations in specific genes may result in an identical clinical manifestation as PAN, such as the deficiency of ADA2 as part of a monogenic disease. Although CPAN predominantly affects the skin, extra-cutaneous symptoms may arise, such as fever, myalgias, arthralgias and neuropathy (mononeuropathy, mononeuropathy multiplex). While the first signs of systemic PAN may be the cutaneous lesions seen in CPAN, upon extensive clinical screening, multi-organ involvement is apparent, particularly in the kidneys, heart, GI tracts and the liver, hence the distinction between the two is essential (27). A comparative clinical study by Alibaz-Oner et al. also concluded that CPAN should be considered as an entirely separate entity from PAN, owing to its chronic relapsing and remitting benign clinical course and little to no mortality (28). While the etiopathogenesis of CPAN is still unclear, it is regarded as a disorder mediated by immune complexes, where direct immunofluorescence often shows C3 and IgM deposition (29). Intriguingly, a study by Kawakami et al. showed a $77.8 \%$ prevalence IgM antibodies targeting the phosphatidylserine-prothrombin complex in patient with CPAN, which would 
then lead to the activation of the classical complement cascade through the production of anti-phosphatidylserine-prothrombin complex antibodies (30). On this note, the connection to prothrombotic processes have also been made, where warfarin therapy has been reported to be effective in the treatment of adult CPAN patients (31).

To date, however, the conceptualization of thrombotic vs. inflammatory processes in LV and CPAN still continues to be perplexing $(31,32)$. 


\section{AIMS}

Distinguishing a skin-limited variant of a vasculitis from its systemic form with a skin component before signs of internal involvement, grants an upper hand in therapeutic management, resulting in a lower risk of potential complications and mortality. To search for indicators in this matter, we examined adult patients with $\operatorname{IgAV}$. IgAV is a small-vessel leukocytoclastic cutaneous vasculitis, often associated with kidney and GI manifestations. Although predictive factors for systemic involvement have been extensively studied in children, there is paucity in the literature regarding adults. NLR is an inflammatory marker, used to assess systemic inflammation in various diseases.

I. Our aim was to evaluate whether NLR can predict and determine the presence and severity of the systemic involvement in adult IgAV patients, hence differentiating skin-limited IgAV from systemic IgAV with a skin component, while also providing prognostic practicality (Study I.)

Though cutaneous biopsy remains an indispensable tool for diagnostic workup in the segregation of vasculitis and vasculopathy, clinical experience leaves us with controversy, without a definite etiopathological line distinguishing the two. LV and CPAN are rare cutaneous diseases of currently unknown etiologies. To date, the concept of thrombosis vs. inflammation in these entities continue to be ambiguous, with varying reports on the effectiveness of anticoagulant and immunosuppressant therapies.

II. Our aim was to assess the presence of vasculopathy in adult patients with CPAN, with the added notion of LV and CPAN being constituents of a clinical evolution of focal vascular occlusion (Study II.) 


\section{MATERIAL AND METHODS}

\subsection{Patients}

Study I. A retrospective review of adult patients diagnosed with IgAV between January 2004 and January 2016 was performed. In accordance with the study criteria used by Takeuchi et al. and Poterucha et al., patients needed to have palpable purpura consistent with the disease, skin biopsy specimen showing leukocytoclastic vasculitis on light microscopy and IgA deposition on direct immunofluorescence $(33,34)$.

Patients were excluded if they had an immunologic comorbidity, coexisting internal malignancy, hematological disorder, cryoglobulinemia or any chronic renal or GI diseases. Additionally, patients who experienced hematochezia, melena or hematemesis two days before or after blood sampling, were also excluded owing to the possibility of neutrophilia being the secondary effect of an acute hemorrhage. The flowchart of patient inclusion is depicted in Figure 1.

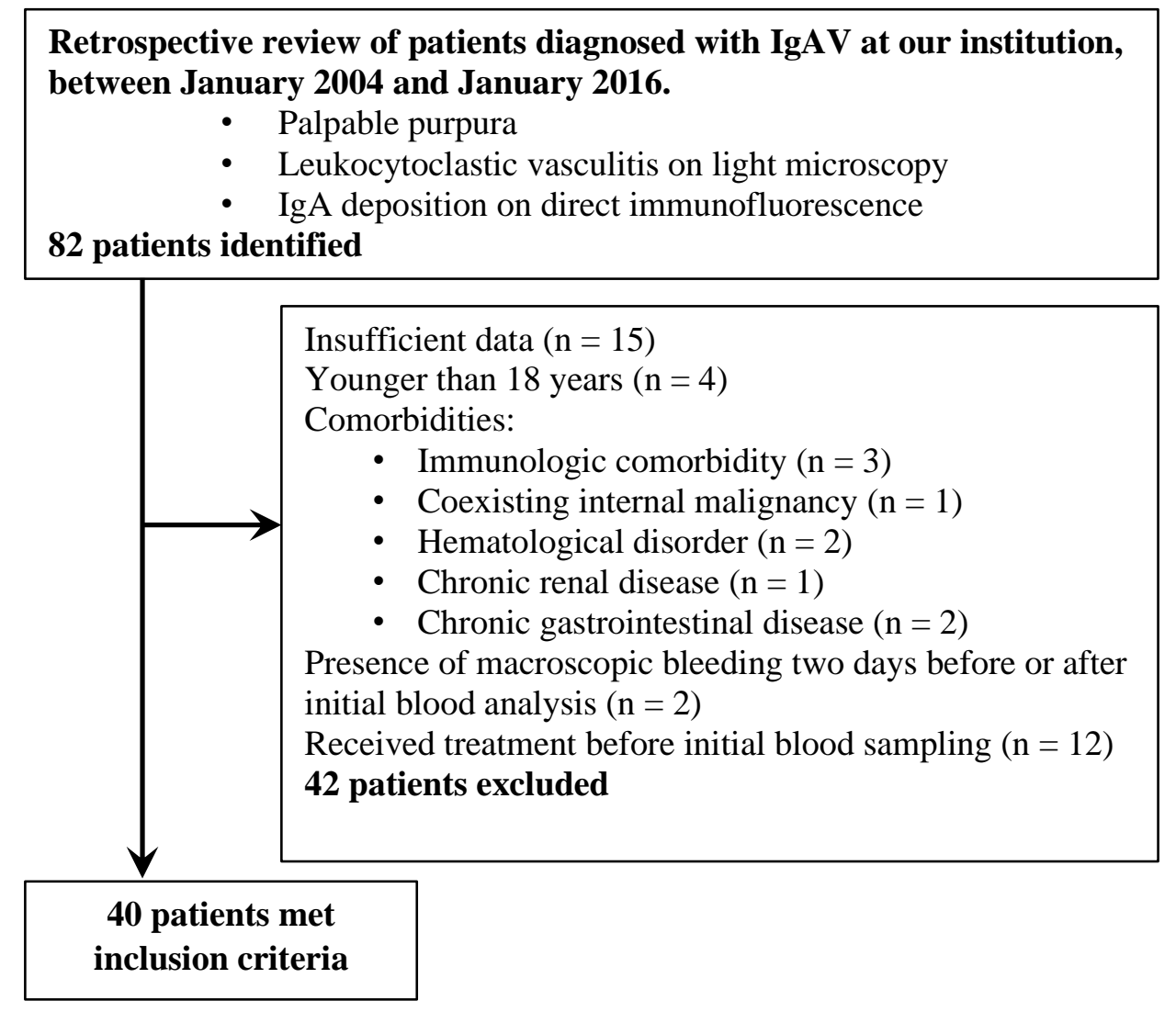

Figure 1. Flowchart of adult IgA vasculitis patient inclusion 
Study II. A retrospective study of eight patients with CPAN was conducted, who were treated at the Department of Dermatology and Allergology, University of Szeged, from 2010 through 2018. The diagnosis of CPAN consisted of histopathological findings of necrotizing vasculitis of the small and medium-sized arteries at the dermal and subcutaneous junction, in addition to presence of clinical manifestations in accordance with CPAN, without any signs suggestive of systemic involvement (1). Cases that did not meet these criteria or lacked clinical information were not included in our report. We then compared our data with those found in the literature regarding the clinical, histopathological and prothrombotic laboratory parameters of $\mathrm{LV}$, to determine whether prothrombotic processes may serve as a common ground in the etiopathology of CPAN and LV.

Both retrospective studies were approved by the local ethics review committee.

\subsection{Data collection}

Study I. For adult patients with IgAV, we analyzed the medical records and registered the following: gender, age, duration of symptoms before blood sampling, clinical symptoms, results of laboratory testing and initial treatment. The laboratory test results included Creactive protein (CRP), erythrocyte sedimentation rate (ESR), white blood cell count (WBC), neutrophil and lymphocyte counts, hematological parameters, urine and stool examinations. NLR was calculated based on the initial complete blood count test, by dividing the neutrophil count with the lymphocyte count. By reviewing the ambulatory records, we also registered any subsequent renal or GI symptoms following initial remission. Patients were followed from baseline visit until loss of follow-up or death.

Study II. Regarding the medical records of CPAN cases, we recorded the age of onset of the disease, gender, duration of symptoms, clinical symptoms, notable medical history with concomitant medications, histopathologic features, initial treatment and laboratory data, which included the immunoserological and thrombophilia screening panel. To further assess the presence or absence of certain cutaneous symptoms of the disease, we revised an electronic photo documentation database, available at our institution, which prospectively enrolls patients.

\subsection{Assessment of GI and renal involvement in IgAV}

We defined GI involvement as clinical signs of hematochezia, melena or hematemesis, or a positive test result for fecal hemoglobin. Renal involvement was determined through hematuria ( $>5$ red blood cells per high-power microscopic field in a centrifuged specimen; 
in the absence of concurrent urinary tract infections, urolithiasis and anticoagulant therapy) or proteinuria (>150 mg/24h), or presence of predominant mesangial IgA deposition on the renal biopsy specimen.

\subsection{Statistical analysis}

Study I. The normality of distribution was analyzed using the Shapiro-Wilk test and parametric or non-parametric statistical tests were used accordingly. Quantitative variables are displayed as mean \pm standard deviation (SD) or with median and interquartile range (IQR). Continuous variables were compared using one-way analysis of variance or KruskalWallis test for multiple groups and independent sample t-test or Mann-Whitney $U$ test for dual groups. Categorical features are summarized with frequency count, percentage and were compared using Fisher's exact test. The intercorrelations between parameters were examined using Spearman's rank correlation coefficient test. A receiver operating characteristic (ROC) curve was performed to examine the prognostic utility of NLR and to identify the optimal cut-off value. Statistical analyses were performed using the Statistical Package for Social Science version 22 (SPSS Inc., Chicago, IL, USA) for Windows. All tests were two-tailed and P-values of less than 0.05 were considered statistically significant. 


\section{RESULTS}

\subsection{Predictors of systemic involvement in adult IgAV patients (Study I.)}

\subsubsection{Basic characteristics of the study sample}

Forty adult patients with IgAV who met the inclusion criteria were identified. The median age was 61 years (range 19-82 years). The cohort included 23 (57.5\%) females and $17(42.5 \%)$ males. Half of the patients had clinical symptoms suggestive of GI and/or renal involvement, of which 6 (15\%) had only renal, 3 (7.5\%) had only GI and 11 (27.5\%) had both renal and GI involvement. Because the presence or absence of arthritis and arthralgia was only recorded in 23 (57.5\%) patients out of the enrolled 40 cases, we have not included this data in the statistical analysis. The median time between the appearance of cutaneous symptoms and initial blood analysis was 9.5 days (IQR 5-14 days). Patients received initial treatment after blood sampling and in all cases either corticosteroid monotherapy or a combination of corticosteroid and antibiotics were employed. Based on the severity of the subsequently developed renal and GI symptoms, therapies were altered accordingly at the discretion of the clinician.

\subsubsection{Clinical features}

As NLR represents an inflammatory response, we also included other routinely used inflammatory markers for comparison. Spearman correlation analysis indicated a significant correlation of NLR with CRP $(\rho=0.482 ; p=0.002)$, ESR $(\rho=0.37 ; p=0.019)$ and WBC ( $\rho=0.469 ; p=0.002)$, however no significant correlation was observed with the duration of symptoms before blood sampling $(\rho=-0.269 ; p=0.094)$ or patient age $(\rho=0.282 ; p=0.078)$, as depicted on Figure 2. To further exclude the possibility of an acute hemorrhage having an effect on NLR, we compared the hematological laboratory values of the enrolled patients based on their organ involvements, which did not indicate a significant difference between the groups (Figure 3).

When stratifying patients based on their renal and GI manifestations and comparing the inflammatory laboratory parameters, there were no statistically significant differences, irrespective of which organ involvement the patients had (Figure 4). Intriguingly, CRP was quite low in those with only GI involvement, which may be due to the small number of patients in this group and their mild clinical manifestations of IgAV. These three patients 

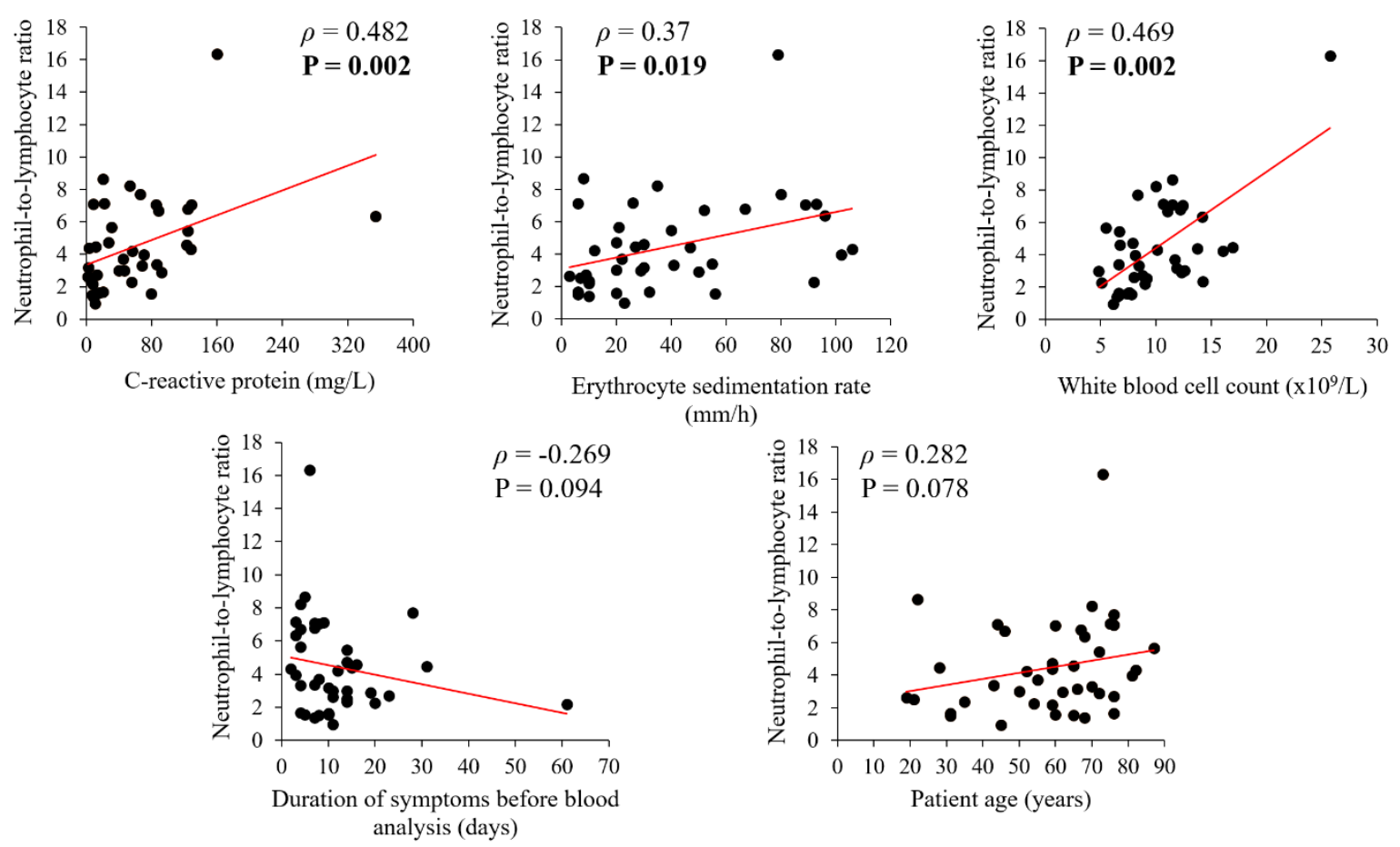

Figure 2. Spearman correlation analysis between neutrophil-to-lymphocyte ratio, other inflammatory markers, duration of symptoms and patient age

did not have extensive cutaneous symptoms and only displayed fecal hemoglobin positivity without any macroscopic bleeding of the GI tract.

Because there were no statistically significant differences in the hematological and the inflammatory values following patient stratification with regards to their organ involvements, the enrolled cases were divided into two groups.
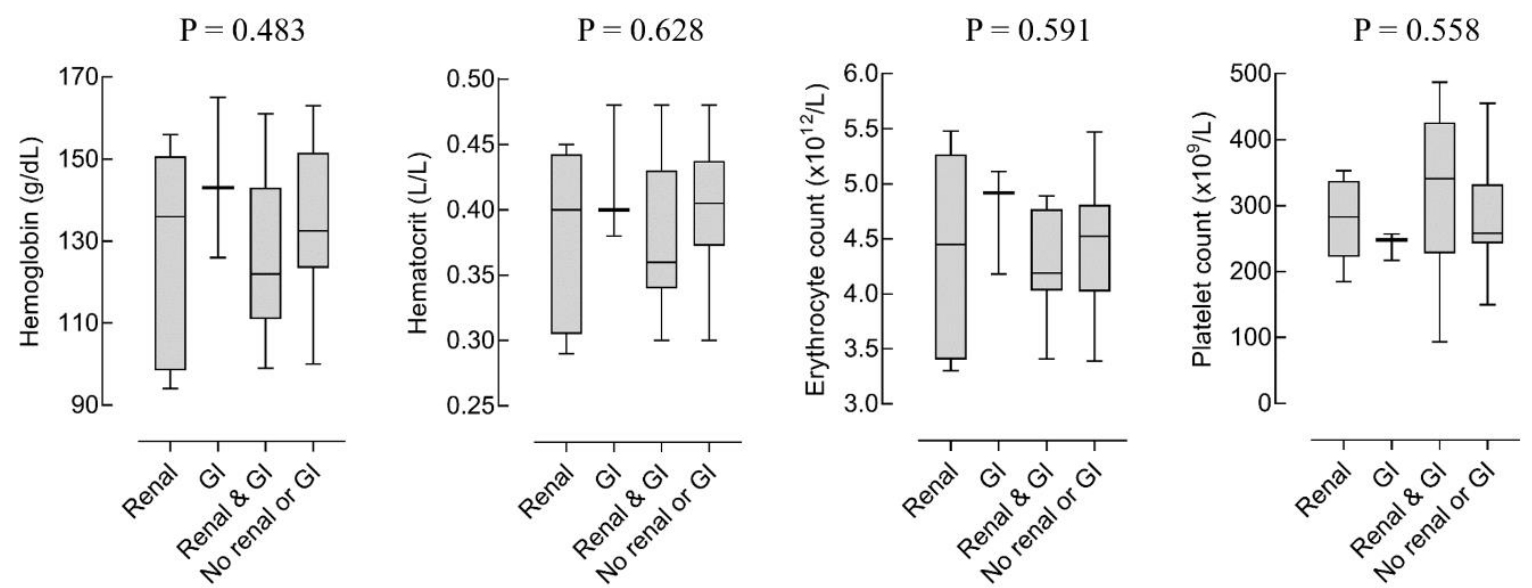

Figure 3. Hematological laboratory values of enrolled patients based on their organ involvements 

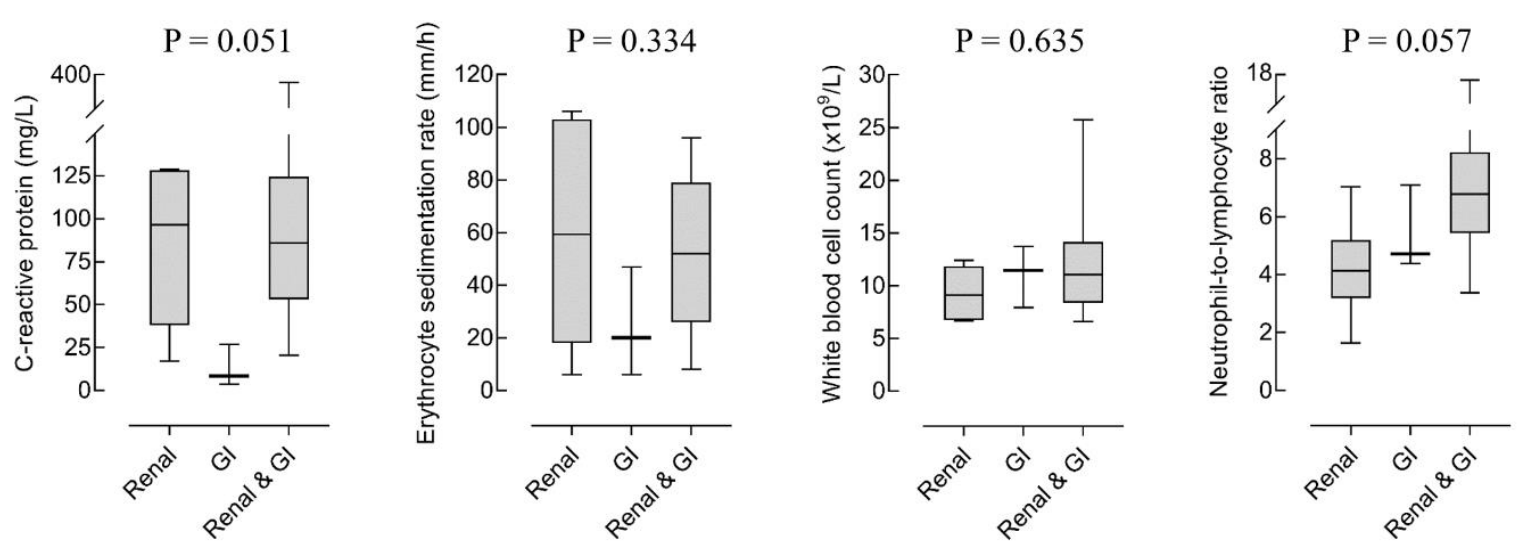

Figure 4. Inflammatory laboratory values of patients based on their organ involvements

While group 1 included patients who only had cutaneous symptoms, group 2 consisted of patients who developed GI and/or renal manifestations of $\operatorname{IgAV}$, in addition to the cutaneous symptoms. The demographic and clinical characteristics of these two groups are detailed in Table 2. Of the registered inflammatory markers, CRP $(p=0.002)$ and NLR $(\mathrm{p}<0.001)$ were significantly higher in group 2 , whereas the other laboratory parameters, age, gender and the duration of symptoms were not statistically associated with systemic involvement (Figure 5).

Six $(30 \%)$ patients in group 2 displayed GI involvement with the presence of macroscopic bleeding, whereas eight (40\%) only had fecal hemoglobin positivity without a clinically apparent hemorrhage. In cases where gross blood was observed, an endoscopic examination was performed, which showed IgAV compatible macroscopic image in all of these patients.

\begin{tabular}{lccc}
\hline Variable & $\begin{array}{c}\text { Group 1 } \\
(\mathbf{n = 2 0})\end{array}$ & $\begin{array}{c}\text { Group 2 } \\
(\mathbf{n = 2 0})\end{array}$ & P-value $^{\mathbf{a}}$ \\
\hline Age; years, mean \pm SD & $56 \pm 20$ & $60 \pm 16.3$ & 0.493 \\
Gender, n (\%) & & & 0.056 \\
$\quad$ Female & $13(65)$ & $6(30)$ & \\
$\quad$ Male & $7(35)$ & $14(70)$ & \\
Duration of symptoms; days, median (IQR) & $11(7-15.3)$ & $7.5(4-12.5)$ & 0.081 \\
Follow-up; months, median (IQR) & $6.8(3.5-24.9)$ & $5.3(2.1-19.1)$ & $\mathrm{NA}$ \\
End stage renal disease, n (\%) & $0(0)$ & $2(10)$ & $\mathrm{NA}$ \\
Death, n $(\%)$ & $0(0)$ & $1(5)$ & $\mathrm{NA}$ \\
\hline
\end{tabular}

'Fisher's exact test was used to compare categorical variables. Student's t-test was used to compare age and Mann-Whitney $\mathrm{U}$ test was used to compare the duration of symptoms and laboratory values.

Table 2. Demographics and clinical features of patients with respect to their systemic involvement 

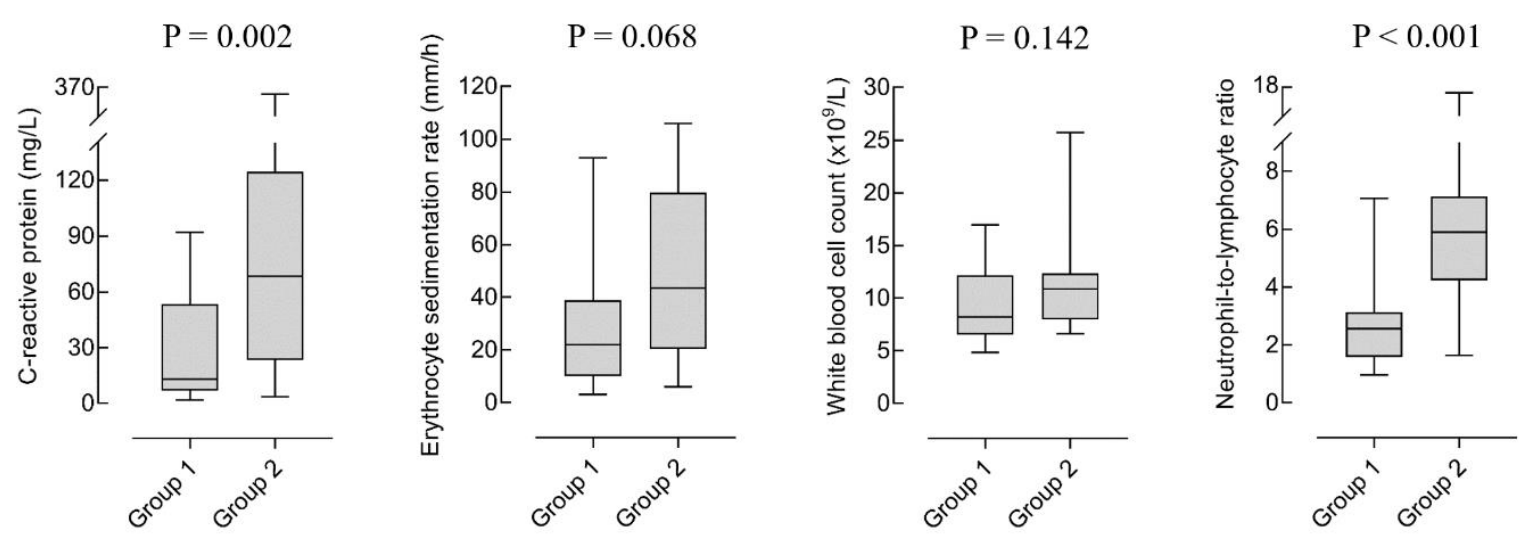

Figure 5. Inflammatory markers of patients with respect to their systemic involvement

With regards to renal symptoms, proteinuria was a frequent finding among those with a systemic involvement $(\mathrm{n}=15 ; 75 \%)$, however none of the patients developed nephrotic syndrome. In two patients, renal involvement progressed into end stage renal disease, 11 and 14 days following initial blood analysis. A kidney biopsy was performed in both cases, which confirmed the association with IgAV. Additionally, these two individuals also developed severe GI bleeding, the former with intussusception and the latter with perforation as a secondary complication, both requiring surgical intervention.

\subsubsection{Receiver operating characteristic curves of NLR versus other inflammatory markers}

ROC curves of NLR and other inflammatory markers in relation to the systemic involvement are depicted in Figure 6. The area under the curve (AUC) for NLR, CRP, ESR and WBC was 0.892 (95\% CI: 0.785-1; p<0.001), 0.779 (95\% CI: 0.635-0.922; $\mathrm{p}=0.003$ ), 0.669 (95\% CI: 0.498-0.839; $\mathrm{p}=0.068$ ) and 0.637 (95\% CI 0.462-0.813; $\mathrm{p}=0.089$ ), respectively. Of the considered laboratory data, NLR provided the strongest diagnostic value, as indicated by the highest AUC value. The optimal cut-off value of NLR for predicting systemic involvement was 3.34 , with a specificity of $95 \%$ and a sensitivity of $85 \%$.

\subsubsection{NLR and disease severity}

We further looked at the correlation of NLR and disease severity in patients with renal and/or GI involvement. For this, we have constructed a simple 7-point scoring system based on the clinical manifestations and course of the disease, observed among our enrolled cases. 


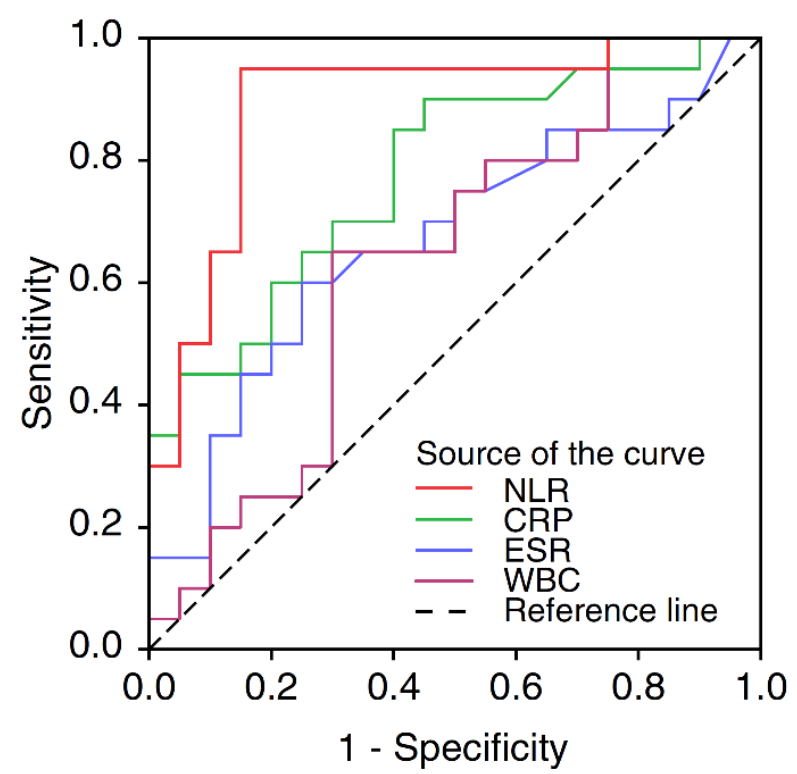

Figure 6. Receiver operating characteristic (ROC) curve of neutrophil-to-lymphocyte ratio versus other inflammatory markers

CRP, C-reactive protein; ESR, erythrocyte sedimentation rate; NLR, neutrophil-to-lymphocyte ratio; WBC, white blood cell count.

Patients received 1 point for each of the following features: hematuria, proteinuria, renal impairment, fecal hemoglobin positivity, macroscopic bleeding from the GI tract, the necessity of intensive care / surgery / dialysis or blood transfusion and death. NLR was found to be significantly correlated with the disease severity score $(\rho=0.51 ; p=0.022)$ (Figure 7).

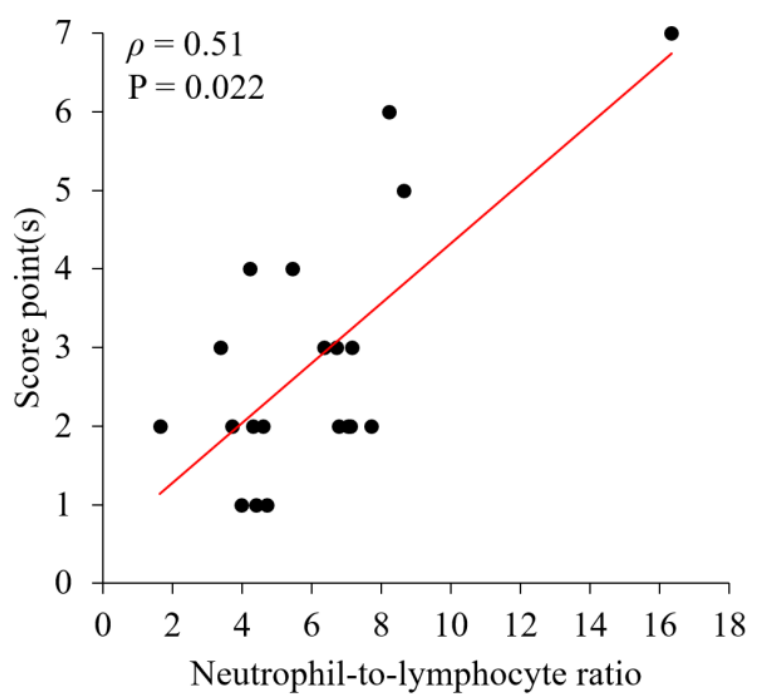

Figure 7. Correlation between neutrophil-to-lymphocyte ratio and disease severity in patients with systemic involvement 


\subsubsection{Assessing the applicability of NLR as a prognostic indicator in clinical practice}

We examined the applicability of NLR in clinical practice by randomly selecting three adult IgAV patients. The clinical presentations of these cases are depicted on Figure 8. The $1^{\text {st }}$ case is a 46-year-old female, who had relatively minor cutaneous symptoms, which developed two days before initial blood examination. Laboratory results showed a CRP level of $88.2 \mathrm{mg} / \mathrm{L}$ and an NLR of 6.7. As NLR was higher than the optimal cut-off value identified in our study, the patient was expected to develop systemic involvement, which she did in the form of proteinuria and colicky abdominal pain with fecal hemoglobin positivity.

The $2^{\text {nd }}$ case is a 54-year-old female, with a twenty-day history of skin lesions prior to initial laboratory examination, whose cutaneous symptoms were much worse than in the case of the $1^{\text {st }}$ patient. Her CRP level was $55.6 \mathrm{mg} / \mathrm{L}$ and NLR showed a value of 2.27. In accordance with her NLR being below the cut-off value, she did not develop any clinically detectable symptoms of systemic involvement, despite having severe cutaneous symptoms.

The $3^{\text {rd }}$ case is a 70-year-old female patient, whose skin symptoms developed seven days before initial blood examination. Likewise, as in the previous case, she had a similar CRP of $53.1 \mathrm{mg} / \mathrm{L}$, but a much higher NLR value (8.23). Four days later, the patient showed signs of severe GI bleeding with intussusception as a secondary complication, requiring surgical intervention. Eleven days later she also developed end stage renal disease.
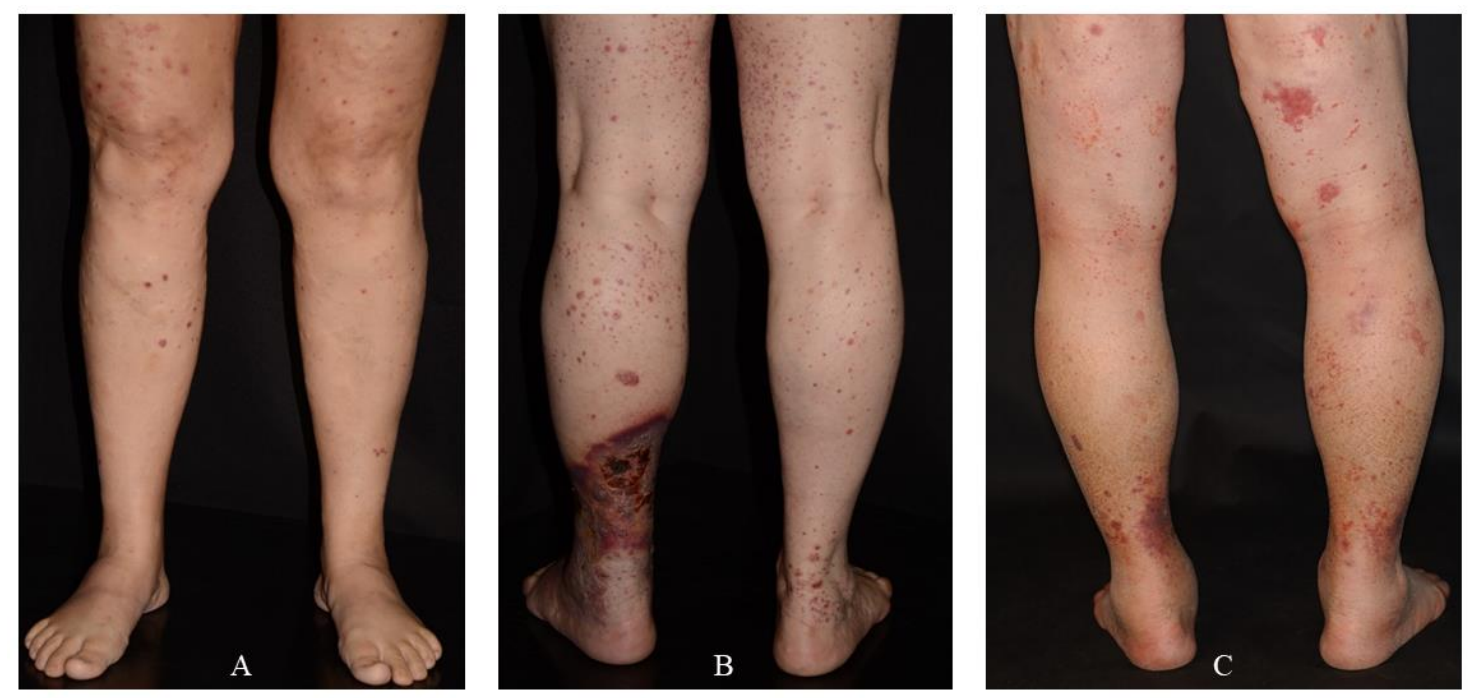

Figure 8. Photo documentation of the clinical presentations of adult patients with IgAV, taken within a day of initial blood examination

Panel A: $1^{\text {st }}$ case; Panel B: $2^{\text {nd }}$ case; Panel C: $3^{\text {rd }}$ case 


\subsection{Assessing the presence of vasculopathy in adult CPAN patients (Study II.)}

\subsubsection{Clinical characteristics}

The clinical characteristics of the 8 patients are summarized in Table 3 . The 5 male and 3 female (M:F ratio, 1.6) patients had a median age of 46 (range, 24-68 years) at diagnosis. The median duration of the relapsing symptoms before cutaneous biopsy sampling was 36 months (range, 2-240 months).

The clinical manifestations consisted of purpura in 4 (50\%), erythematous papules or plaques in $7(87.5 \%)$, subcutaneous nodule in $1(12.5 \%)$, livedo racemosa in $7(87.5 \%)$, cutaneous ulcer in 7 (87.5\%) and atrophie blanche in 7 (87.5\%) cases. Without exception, the skin symptoms were always localized to the lower extremities, with notable predilection for the ankle regions. Notably, two of the patients (25\%) also had peripheral neuropathy without motor deficit localized to the lower extremities, attributable to CPAN. Muscle-nerve biopsies in these instances were not performed. With regards to known comorbidities, 2 (25\%) patients had hypertension, of which one also had atrial fibrillation and was on apixaban treatment at the time of CPAN diagnosis. All cases were screened for antinuclear antibodies, ANCAs and complements, with negative results.

\subsubsection{Histological features}

In all cases, deep skin biopsies containing the subcutis were obtained. Each specimen displayed hyalinized dermal blood vessels, perivascular neutrophilic and lymphocytic infiltrate, leukocytoclasia and marked narrowing of the vessel lumens (Figure 9). Notable thrombosis was not apparent in most cases (87.5\%), with only one specimen displaying extensive thrombosis in the papillary and deep dermis. Fibrinoid deposition in the papillary and deep dermal vessels was present in $7(87.5 \%)$ patients. Direct immunofluorescent staining showed complement deposition in the vessel walls in $8(100 \%)$ cases, with IgA in $4(50 \%)$ and $\operatorname{IgM}$ in $4(50 \%)$ cases.

\subsubsection{Coagulation disorders}

Procoagulative conditions were found in $7(87.5 \%)$ patients (Table 3). Elevated levels were detected of fibrinogen in $2(25 \%)$, antithrombin III in $2(25 \%)$, homocysteine in 1 (12.5\%), factor VIII in $3(37.5 \%)$ cases. Protein S deficiency was detected in one (12.5\%) patient and the lupus anticoagulant screening test was positive in $3(37.5 \%)$ cases, without increased values in anti-cardiolipin or beta-2-glycoprotein antibodies. 


\begin{tabular}{|c|c|c|c|c|c|c|c|c|c|c|}
\hline 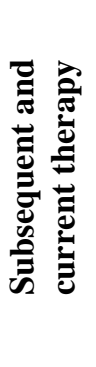 & & 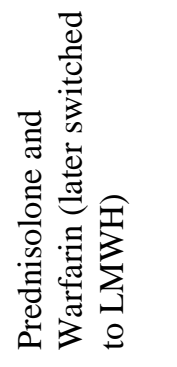 & 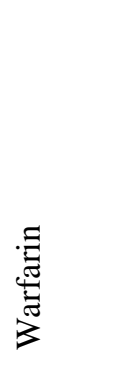 & 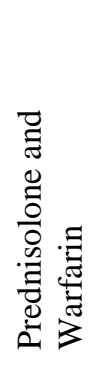 & 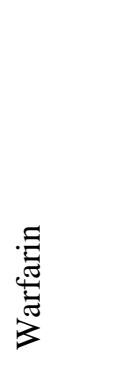 & 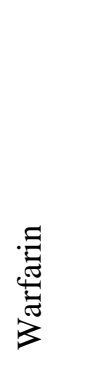 & 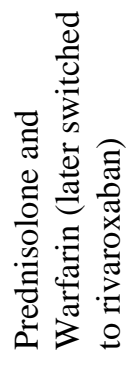 & 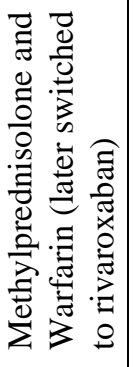 & 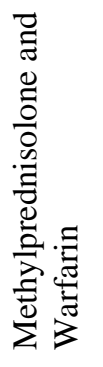 & 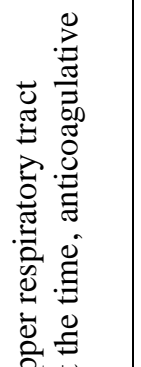 \\
\hline 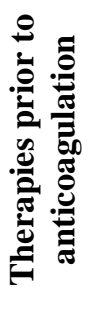 & & 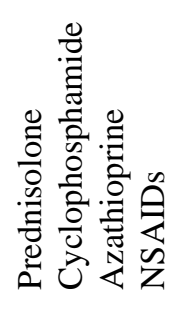 & 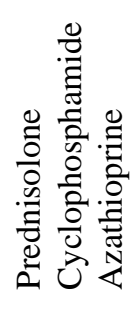 & 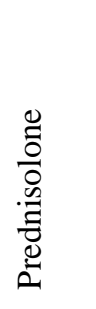 & 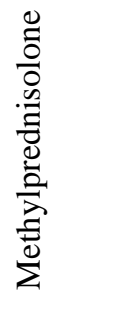 & 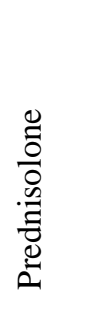 & 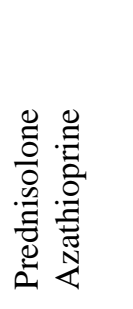 & 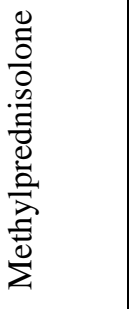 & 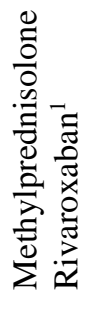 & 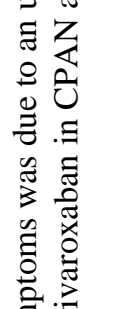 \\
\hline 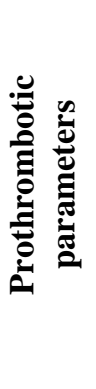 & & 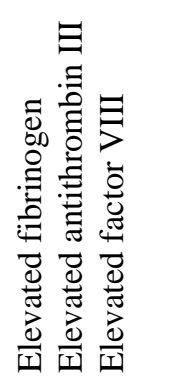 & 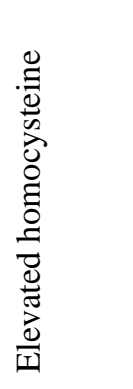 & 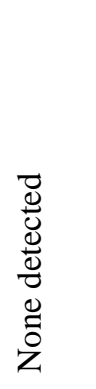 & 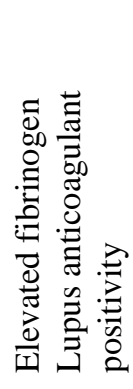 & 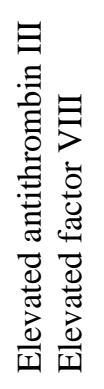 & 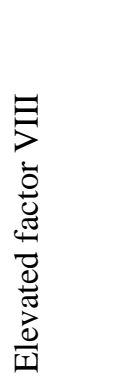 & 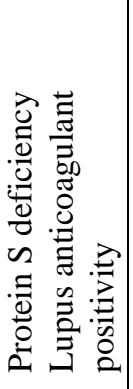 & 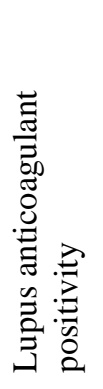 & 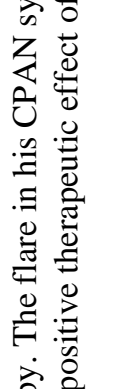 \\
\hline \multirow{7}{*}{ 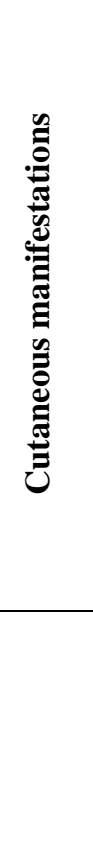 } & $\begin{array}{l}\text { Atrophie } \\
\text { blanche }\end{array}$ & + & + & + & + & + & + & + & ' & \multirow{7}{*}{ 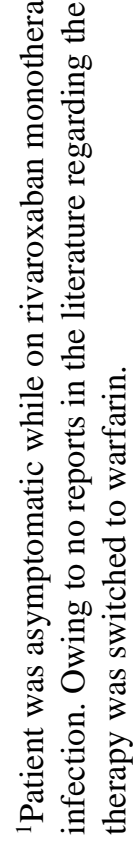 } \\
\hline & Ulcer & + & + & + & + & + & ' & + & + & \\
\hline & $\begin{array}{c}\text { Livedo } \\
\text { racemosa }\end{array}$ & + & + & + & + & + & + & + & ' & \\
\hline & $\begin{array}{l}\text { Subcutaneous } \\
\text { nodule }\end{array}$ & ' & ' & ' & ' & ' & + & ' & ' & \\
\hline & $\begin{array}{c}\text { Erythematous } \\
\text { papule / } \\
\text { plaque }\end{array}$ & + & + & ' & + & + & + & + & + & \\
\hline & Purpura & + & + & ' & ' & ' & 1 & + & + & \\
\hline & 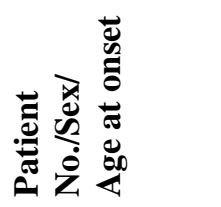 & $\sum_{\substack{\infty \\
\Sigma}}^{\infty}$ & 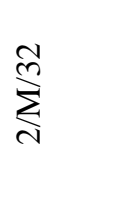 & 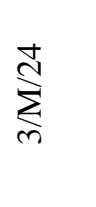 & 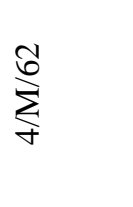 & $\underset{⿱ m}{\stackrel{m}{n}}$ & $\frac{O}{+\infty}$ & $\underset{\substack{+ \\
\text { I }}}{\frac{1}{r}}$ & $\sum_{\infty}^{\infty}$ & \\
\hline
\end{tabular}

Table 3. Clinical features and employed therapeutic modalities in patients with cutaneous polyarteritis nodosa.

M, male; F, female; minus sign, absent; plus sign, present; LMWH, low molecular weight heparin. 

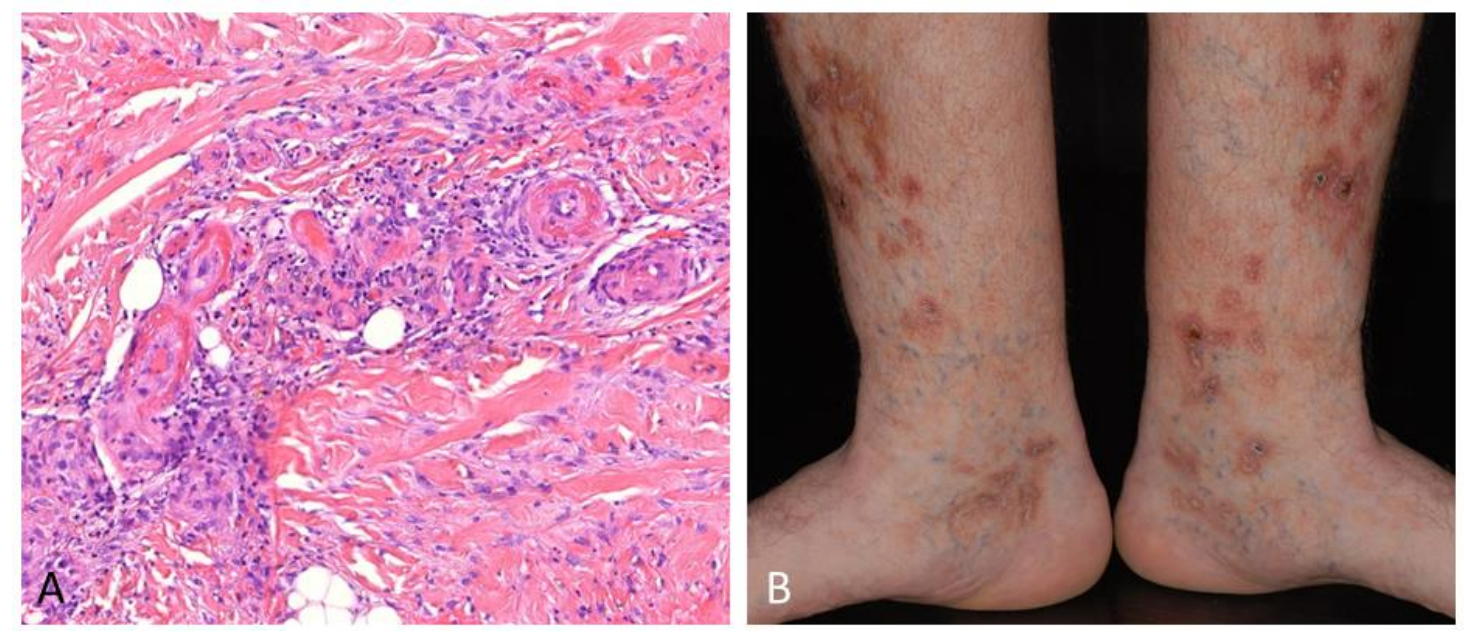

Figure 9. Histopathological and clinical image of patient No 2.

Panel A: Histopathology image showing segmental fibrinoid necrosis of a blood vessel, with marked perivascular neutrophilic and lymphocytic infiltrate, in addition to fibroelastic vessel wall thickening, causing narrowing of the lumen and focal vascular occlusion (hematoxylin and eosin staining); Panel B: Corresponding clinical image at the time of cutaneous biopsy sampling.

\subsubsection{Therapeutic regimen}

Initial therapies consisted of corticosteroids, however $3(37.5 \%)$ patients required alteration in their immunosuppressants within a median of 4 months (range, 1.3-4.6 months; Table 3). Subsequently, only partial response was achieved long-term (median, 12 months; range, 7.2-58.1 months), thus, in consideration of the procoagulative parameters, warfarin therapy was initiated in all cases. All patients responded favourably to anticoagulation (case example illustrated on Figure 10). Three cases remained asymptomatic with warfarin monotherapy alone.
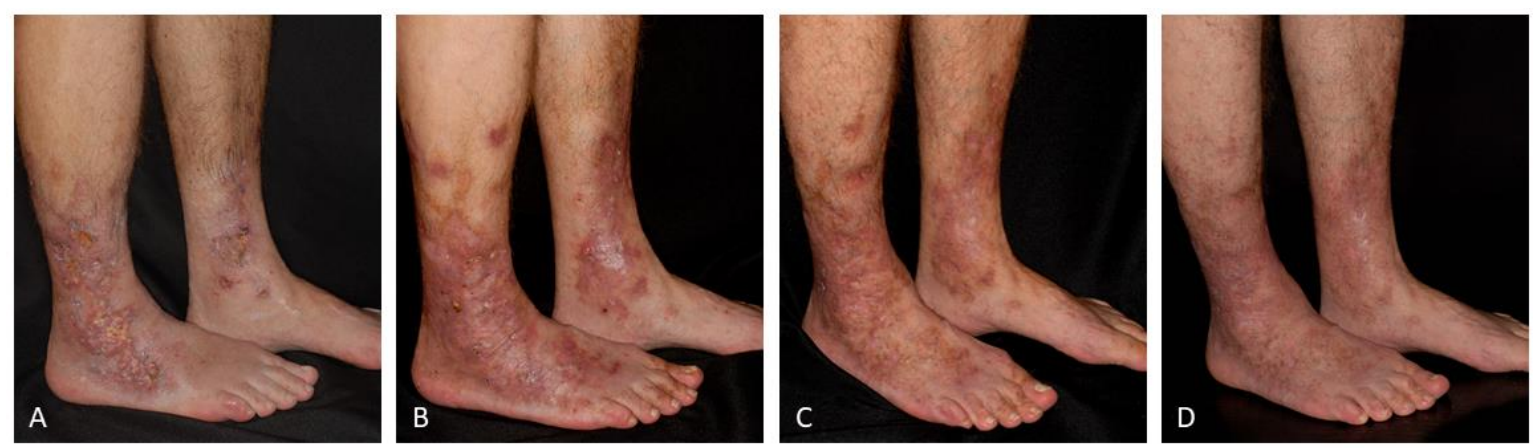

Figure 10. Clinical images of patient No. 1 with cutaneous polyarteritis nodosa, depicting the improvement of skin symptoms following the addition of warfarin therapy to prednisolone.

Panel A: Unresponsive cutaneous ulcerations following multiple immunosuppressant modalities.

Skin symptoms after Panel B: 1 month, Panel C: 7 months, Panel D: 12 months, following the initiation of warfarin therapy. 


\subsection{Difficulty in the segregation of vasculitis and vasculopathy in clinical practice}

The proper distinction between vasculitis and vasculopathy is further challenged by the heterogenous pattern observed in each individual patient, comprised of environmental factors, genetics, comorbidities and their concurrent therapies as well as the current stage of the disease evolution. With a complex case detailed below, we wish to exemplify such a dilemma, from a clinician's point of view.

The present case describes a 49-year-old woman who had been diagnosed and treated with seropositive and anti-mutant-citrullinated vimentin antibody (MCV) positive rheumatoid arthritis (RA) for 13 years. Intriguingly, she also had intermitting cutaneous ulcerations on both legs in the previous 6 years, which were not characteristic for either LV nor CPAN and were not preceded with inflamed nodules. A skin biopsy was performed at the time, which showed leukocytoclastic vasculitis with IgM and C3 deposition, however, the patient never had purpuric lesions. Notably, perinuclear ANCA with indirect immunofluorescence and myeloperoxidase antibody with enzyme-linked immunosorbent assay were detected once, but no other symptoms specific of an ANCA-associated vasculitis were present. Antinuclear antibodies and anti-dsDNA were negative. In view of the high titer rheumatoid factor and anti-MCV-positivity, the presence of rheumatoid nodules, interstitial lung disease, pericarditis and mononeuritis, the cutaneous vasculitis was considered to be an extra-articular manifestation of RA.

In 2009, thrombosis occurred in her upper right arm. Extensive clinical and laboratory examination with thrombophilia screening revealed heterozygote factor V Leiden mutation and the patient was put on acenocoumarol treatment. Retrospectively, this procoagulative state may have also played a major role in the development of the cutaneous ulcerations and their accompanying constant pain.

She had previously been treated with varying doses of corticosteroid for 6 years, chloroquine, leflunomide, azathioprine and methotrexate; received cyclophosphamide (500mg) pulse therapy on 7 occasions, followed by adalimumab (40mg sc biweekly) for 15 months. Tocilizumab (TCZ) is a humanized anti-human interleukin-6 (anti-IL-6) receptor antibody, which is used with success in the treatment of RA. Anti-IL-6 biologic therapy was necessitated by the subsequent increase in her disease activity and TCZ was intravenously initiated in June 2013. Over the course of the next 19 months, the patient received 15 TCZ 
infusions $(8 \mathrm{mg} / \mathrm{kg})$, methotrexate $(15 \mathrm{mg} /$ week) for 17 months and maintained a low dose of corticosteroid therapy (reduced from the initial $12 \mathrm{mg} /$ day to $4-2 \mathrm{mg} /$ day) for 14 months. During this time, her arthritis was inactive and the cutaneous ulcerations, due to her immunocomplex leukocytoclastic vasculitis, showed moderate improvements.

She received her 16th TCZ treatment as a subcutaneous injection (162mg), in order to achieve a more consistent distribution of the medication. Three days following treatment, the patient experienced severe pain and burning sensation of the skin and was presented with multiple palpable purpuras with central necrotic areas mainly on the legs, buttocks and lower torso (Figure 11. A, B).

Initial blood analysis showed minor C-reactive protein $(7.2 \mathrm{mg} / \mathrm{l}$, normal value $<5 \mathrm{mg} / \mathrm{l})$ elevation, increased D-dimer $(15 \mu \mathrm{g} / \mathrm{ml}$, normal value $<0.5 \mu \mathrm{g} / \mathrm{ml})$ levels and normal white blood cell count $\left(6.5 \times 10^{9} / 1\right.$; neutrophils $\left.63.9 \%\right)$. The patient had an INR value of 0.94 , despite being on acenocoumarol treatment at the time. Liver and kidney functions were normal. Chest X-ray revealed bilateral interstitial reticular opacities. The patient did not have any clinical symptoms suggestive of pneumonia or abdominal pain and faecal occult blood testing was negative. Skin biopsy from a purpuric lesion revealed leukocytoclastic vasculitis with novel features of extensive intraluminal thrombosis and fibrinoid necrosis (Figure 11. C, D, E). Direct immunofluorescent staining of the specimen showed deposits of IgG in addition to $\operatorname{IgM}$ and $\mathrm{C} 3$.

Because her cutaneous symptoms rapidly progressed and resembled lesions often seen in vasculopathies such as purpura fulminans, the patient was given two units of fresh frozen plasma on three occasions and received $125 \mathrm{mg}$ corticosteroid parentally, to which a marked improvement could be observed. Additionally, acenocoumarol was switched to enoxaparin. Subsequent laboratory results showed decrease in D-dimer $(5.15 \mu \mathrm{g} / \mathrm{ml})$ values. Treatment proved to be efficient and the cutaneous lesions gradually ameliorated. As there were no newly administered medications, nor any recent infections, we concluded that the severe adverse skin symptoms were most likely induced by the most recent subcutaneous TCZ injection, thus further anti-IL-6 treatment was discontinued. Subsequently, the patient did not develop any purpuric lesions or thrombotic events throughout the following years. 

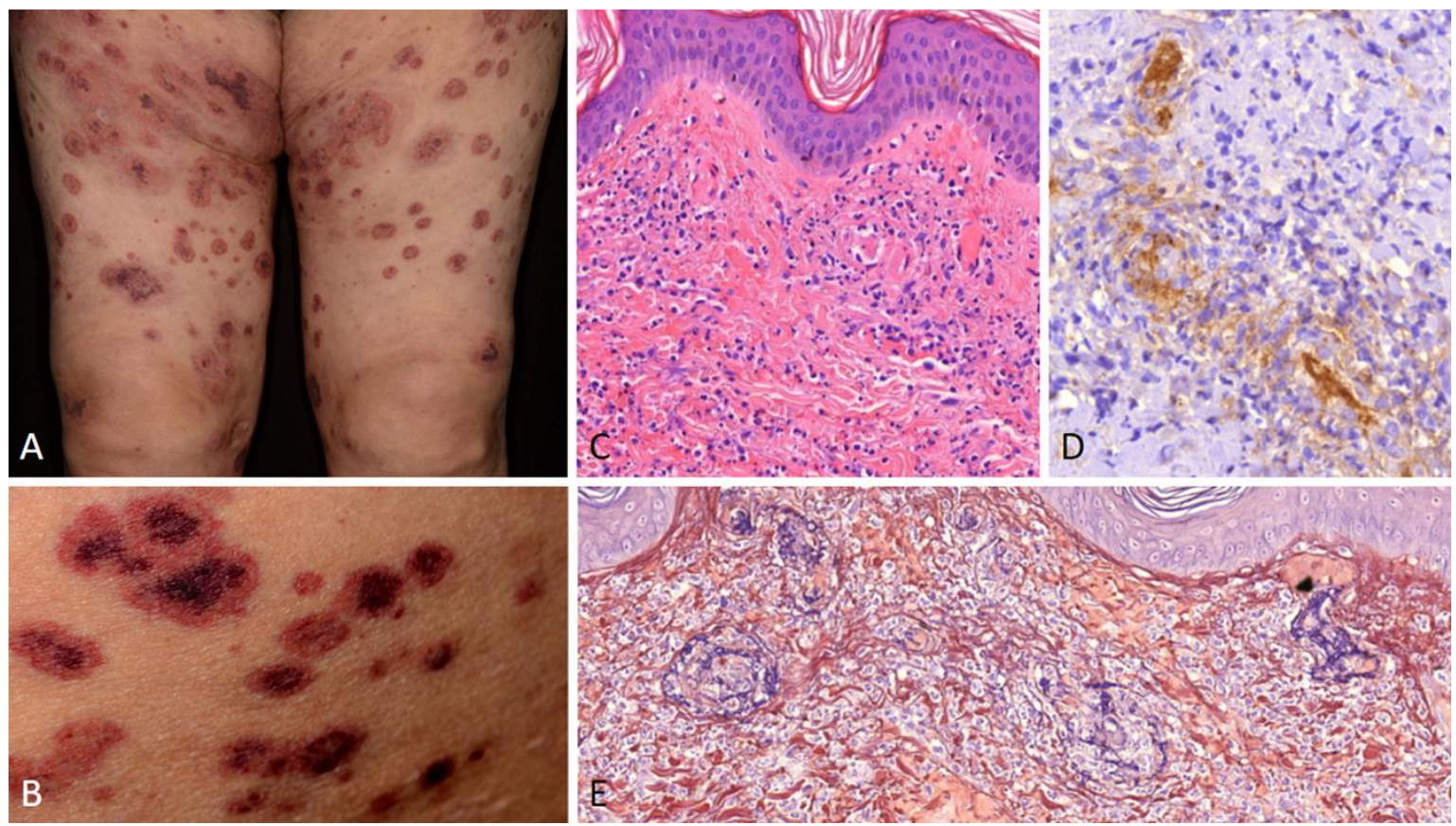

Figure 11. Purpuric lesions in a patient with rheumatoid arthritis after three days of subcutaneous tocilizumab injection.

Panel A, B: Multiple purpuric lesions with central necrotic areas on the thighs; Panel C: Light microscopic image showing leukocytoclastic vasculitis with erythrocyte extravasation; Panel D: Factor VIII immunohistochemical staining showing multiple thrombi in the blood vessel lumen;

Panel E: PTAH staining of the specimen depicting multiple small blood vessels with signs of extensive fibrinoid necrosis. 


\section{DISCUSSION}

Although IgAV is often a self-limiting and benign disease in children, severe complications may occur in adults, including renal impairment and serious GI bleeding, requiring intensive care or surgery $(9,35)$. To date, no distinct differentiator can be employed in the stratification of skin-limited IgAV from a systemic IgAV with a skin component. As such, in accordance with current concepts, the two are considered to be on the same disease spectrum. A number of studies have been conducted with the aim to correlate various clinical features with systemic involvement in adults, such as direct immunofluorescence findings of skin biopsy specimens and skin lesion distribution, though the results are dissenting $(33,34,36)$. Additionally, endoscopic features of GI lesions and histopathological findings of renal biopsies have also been studied, however these modalities can cause serious complications and may be contraindicated in certain cases $(37,38)$. Therefore, in order to gain more prognostic information, we examined the utility of a noninvasive and easily obtainable cost-effective laboratory parameter. To our knowledge this is the first biopsyproven case-control study to investigate the predictive value of NLR for systemic involvement in adult IgAV patients.

Half of the patients included in Study I. developed renal and/or GI involvement, of which the majority concurrently had both organs affected. In accordance with this finding, other studies have also noted high incidence of simultaneous renal and GI manifestations in adult IgAV $(38,39)$. It should be mentioned however, that some researchers could not confirm this finding, which may be related to the varying criteria employed for defining systemic involvement $(33,40)$. Male gender has also been previously described as a poor prognostic indicator for systemic involvement, however our results did not reach statistical significance $(9,39)$.

When stratifying patients based on their renal and GI involvement and comparing the registered laboratory results, there were no significant differences in the hematological and the inflammatory laboratory values, which implies that NLR and the other inflammatory markers are not organ specific prognostic indicators and their elevated values were not the secondary effect of an underlying severe bleeding. Consequently, the high NLR observed in our study, is therefore likely to be the result of an inflammatory response.

Our results demonstrated that out of all the considered inflammatory parameters, NLR had the strongest prognostic value. The optimal cut-off point for predicting systemic 
involvement was 3.34 with a specificity of $95 \%$ and a sensitivity of $85 \%$. Additionally, we also found that pretreatment NLR values significantly correlated with the severity of the disease in patients who developed systemic involvement. It is important to note however, that when constructing the scoring system used to evaluate this correlation in our study, we only considered the presence or absence of certain clinical manifestations, rather than assessing the extent of renal or GI symptoms on a spectrum. In medical literature, the applicability of NLR in disease severity scoring is not new however, as it has been demonstrated viable for the measurement in the following scoring systems in patients with sepsis: Sequential Organ Failure Assessment, Simplified Acute Physiology Score III and Acute Physiology, Age, Chronic Health Evaluation $(41,42)$.

Progression to end stage renal disease was observed in two individuals. Compared to children, adult IgAV patients are more likely to present with a delayed renal involvement, often requiring close monitoring and diligent testing for even up to 6 months following the onset of $\operatorname{IgAV}$, despite favorable initial laboratory results (43). While delayed renal involvement was not observed in any of our cases, it should be noted that 20 patients (10 individuals from group 1 and 10 individuals from group 2) did not have a follow-up time period of at least 6 months. Although one of the patients with a lower NLR than the cut-off value identified in our study developed renal manifestations of $\operatorname{IgAV}$ (in the form of microscopic hematuria and proteinuria) during his admission, he remained asymptomatic throughout the follow-up time period (9 years).

More than a third of our patients had GI involvement, which is in accordance with previous reports $(9,10)$. In most cases, the symptoms consisted of colicky abdominal pain with fecal hemoglobin positivity, however some patients developed macroscopic bleeding. Severe complications, such as intussusception and perforation were also observed in two cases, the latter resulting in a fatal outcome. None of the patients in our cohort with a lower NLR value than 3.34 displayed any clinical signs suggestive of GI involvement, during their admission and follow-up, which further highlights the prognostic value of NLR. Although a recent study demonstrated the applicability of this ratio with regards to GI bleeding in adult patients with cutaneous vasculitis, the employed criteria for the diagnosis of IgAV did not include skin histopathology examination (44). In consideration of the probable clinical overlap between hypersensitivity vasculitis and $\operatorname{IgAV}$, differentiating between these two entities is imperative when assessing and predicting renal or GI involvement (45). Consequently, the clinical significance of the presence or absence of $\operatorname{Ig}$ A deposition found 
by direct immunofluorescence of the cutaneous biopsy specimen should not be overlooked, as it enhances the diagnostic specificity $(4,46,47)$.

The significance of our findings is limited by the retrospective nature of Study I. and its single-center sample size. Nevertheless, as our selection was based on both clinical and histopathological findings, with the exclusion of patients with other background diseases, we believe our data represent accurate observations. Larger prospective multi-center studies are required to validate our findings and to establish a generally acceptable cut-off value of this ratio. The routine testing for qualitative blood count in clinical practice at minimal cost and little-to-no risk to the patient makes NLR a promising measurement for systemic inflammation. Whether the viability of this biomarker permits the development of prospective therapeutic studies, remains to be determined. Nonetheless, there seems to be reasonable potential for this marker as a tool for clinical risk stratification at the time of IgAV diagnosis, thus also allowing for a more patient-specific therapeutic intervention.

On the matter of the vasculitis and vasculopathy conundrum, we examined the clinical features of a single-organ vasculitis of the skin, focusing on the presence of procoagulative components of the disease.

Presently, CPAN is considered to be a necrotizing vasculitis and although its etiology is not fully elucidated, current concepts advocate that it is mediated by immune complex deposition (29). Likewise, the pathogenesis of LV also lingers on uncertain grounds, though, the interpretation of non-inflammatory thrombosis of the dermal vessels remains to be widely accepted (25).

Contrariwise, reports of LV and CPAN not responding to the conventional treatments, leave us with controversy regarding the aforementioned concepts $(31,48)$.

Additionally, despite the presumed difference in their underlying pathomechanisms, these two entities share notable clinical similarities not only in their cutaneous manifestations but also in their recurrent and usually non-progressive clinical courses, implicating a link amongst their pathophysiological mosaics.

In Study II. the cutaneous manifestations of CPAN were compatible with LV in all cases, with pain being a constant subjective symptom. Intriguingly, despite the vast majority of the literature highlighting the presence of subcutaneous nodules, only one patient displayed such lesions upon presentation. Contrarily, the pathologic form of livedo 
reticularis, also regarded as livedo racemosa, was present in all but one of the cases. While livedo reticularis can develop due to vessel wall pathology (when medium-sized arterioles of the dermal-subcutaneous layer are affected), it may also be a sign of intraluminal pathology, either owing to a slowed intravascular blood flow or complete obstruction of the vessel lumen. As a result, the cutaneous zone of the venous predominance is affected, causing the dilation of the cutaneous venules and accumulation of deoxygenated blood in the venous plexus, leading to the clinical presentation of the characteristic net-like pattern (49). Obstruction can result from either an emboli (e.g. cholesterol emboli) or thrombosis, thus linking it to hypercoagulative states, such as antiphospholipid syndrome, protein $\mathrm{C}, \mathrm{S}$ or antithrombin III deficiency. In LV, a combination of both vessel wall pathology and intraluminal obstruction is postulated which is also in line with CPAN. Atrophie blanche was a prominent feature in the majority of cases. For an extended period of time, this term was used as a diagnostic descriptor for livedoid vasculitis, which was later revised as LV, denoting the presence of blood vessel lumen obstruction, seen as segmental hyaline degeneration of the vessel wall, endothelial proliferation and deposition of fibrin in the lumen $(50,51)$. Additional manifestations of the series included peripheral neuropathy in two of our patients, which has also been described in LV, thought to be the result of the thrombotic processes affecting the vasa nervorum $(24,52)$. No evidence of autoimmune abnormalities were detected in our series, thus excluding other forms of vasculitis or rheumatologic diseases.

Histological features of our study sample displayed segmental hyalinizing vasculitis, with intravascular deposition of fibrin and secondary changes deriving from vascular occlusion. Perivascular lymphocytic infiltrate, in addition to neutrophils, was a prominent feature in our cases, which is also often seen in LV (53). Albeit extensive intraluminal thrombosis, characteristic of LV, could only be seen in one of our cases, it is commonly described in CPAN alike (54). Whilst LV initially appears with a histopathologic picture showing fibrin thrombi without evidence of inflammation, it is later presented as hyalinized fibrin rings with lymphocytic infiltrate, signs of vasculitis and erythrocyte extravasation, accompanied by neutrophilic infiltrate and immunoglobulin deposition $(21,23,55)$. As the duration of symptoms before biopsy sampling was protracted in our series, it further underscores the concept of disease evolution as deciphering primary and secondary histopathologic features remain to be challenging. Additionally, while superficial skin histopathology may show vessel occlusion identical to LV, they are secondary changes to 
the inflamed vessels in the deep dermis, thus, superficial biopsies can be misleading in CPAN, which may also explain the discrepancy in the literature (56). This further underscores the importance of deep cutaneous biopsies and may also accentuate the necessity of repeated excisional biopsies to reveal the true underlying pathology. LlamasVelasco et al. further emphasizes the interrelation between the two by demonstrating LV and CPAN coexisting within the same skin biopsy specimen (53).

Similar to a hypercoagulable state seen in LV, we found coagulopathies in the majority $(87.5 \%)$ of our patients, giving a considerable role for anticoagulant therapy in CPAN as well. While immunosuppressives are the mainstay, long-term management of our patients necessitated the introduction of anticoagulative treatment, with good therapeutic response $(31,32)$. Although cutaneous biopsy provides essential morphological insights, it is also important to consider the clinical experience and therapeutic responses, hence, we believe a considerable role should be given to the vasculopathic aspects of CPAN, in addition to vasculitis, which may serve as a common ground for widening our scope of the vasculopathy vs. vasculitis question.

As a limitation of this retrospective study on such a rare disease, we acknowledge the small sample population, however, in consideration of the continued controversy regarding thrombosis vs. inflammation, our endeavour was to propose a new approach to the matter in question by affiliating LV with CPAN in the context of constituents of a clinical evolution: a changing face of focal vascular occlusion.

Lastly, the accentuation of thrombosis and inflammation going hand-in-hand must be noted as more evidence comes to light regarding the intricate connection between the innate immunity and the coagulative cascade, a pathomechanism which has been termed immunothrombosis (57). Immune-mediated thrombosis is thought to be an early stepping stone in the evolution of the immune system, where the innate immune cells developed prothrombotic pathways as a form of protection against pathogens in the vasculature by thrombi formation to capture and limit the spreading of pathogens (58). The basic principles behind this response are the activation of monocytes, neutrophils and their interaction with the coagulation cascade. These innate immune cell specific pathways serve as potentiators for thrombosis, which are activated by certain stimuli, such as the presence of pathogenassociated molecular patterns or damage-associated molecular patterns. When activated, monocytes are able to release tissue factor expressing microparticles, which then activate the 
extrinsic coagulation pathway, while neutrophils are capable of releasing neutrophil extracellular traps (NETs), which are comprised of DNA matrix, harboring histones, nucleosomes and neutrophil elastase (Figure 12) $(59,60)$. NETs are considered to be crucial structural factors in the formation of immune-elicited thrombi, as they promote the intrinsic coagulation pathway by activating factor XII, as well as directly initiating fibrin formation (57). Previous studies have also demonstrated that these processes occur in autoinflammatory disorders, in the absence of microbial pathogens, such as in ACNAassociated vasculitides (61-63). Owing to these mechanisms, increased prevalence of thrombosis has been observed in several other autoimmune diseases (64).

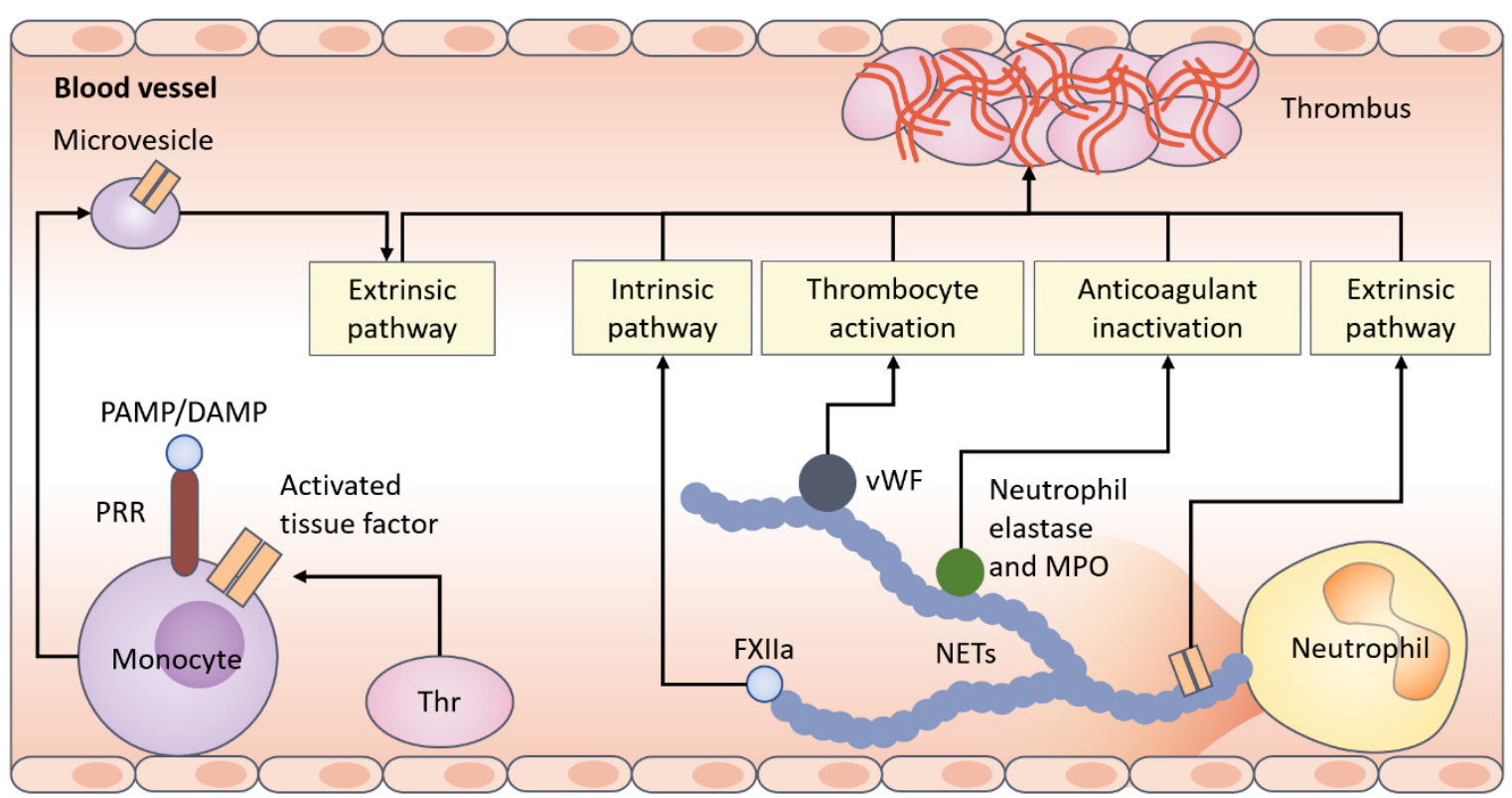

Figure 12. The basic principles of immunothrombosis

DAMP, damage-associated molecular patterns; FXIIa, activated factor XII; NETs, neutrophil extracellular traps; MPO, myeloperoxidase; PAMP, pathogen-associated molecular patterns;

PRR, pattern-recognition receptor; Thr, thrombocyte; vWF, von Willebrand factor

Reproduced from reference (57).

The described complex case of RA with TCZ-associated adverse reaction poses a number of questions regarding the possible underlying pathomechanisms. On the matter of an inflammation driven process, most biologic-agent-induced vasculitis case reports are associated with antitumor necrosis factor- $\alpha$ (anti-TNF- $\alpha$ ) therapies. Although their pathophysiology is still poorly understood, probable mechanisms have been proposed such as the formation of anti-TNF- $\alpha$-TNF- $\alpha$ immunocomplex which results in type III hypersensitivity (65). It may be plausible that TCZ can induce vasculitis through a similar 
process, by binding to soluble IL-6 receptors and creating immune deposits in the vasculature, which process may be supported by the presence of $\mathrm{IgG}$ deposition found in the direct immunofluorescence specimen of the present case, considering that TCZ is a humanized IgG1 class monoclonal antibody (66).

On the other hand, it may also be reasonable to consider that TCZ was not part of the immunocomplex but was perhaps involved in an indirect way. Compared to anti-TNF- $\alpha$ therapies, only a few cases with TCZ-induced immune disorders have been described, which might also suggest a lower immunogenicity or a prerequisite of specific factors which then promote susceptibility $(67,68)$. It is of interest that the presented skin aberrations manifested so profoundly. In our patient, the main engrossing histopathological findings were the significant amounts of thrombi in the cutaneous vasculature, seen in vasculopathies. In light of the markedly elevated D-dimer, a thrombogenic effect was responsible for the severity of this reaction. IL-6 plays an important role in the pathogenesis of autoimmunity and also facilitates blood coagulation through various pathways $(69,70)$. It is possible that administration of TCZ could have increased serum IL-6 levels due to the antibodies binding to IL-6 receptors leading to thrombosis in the cutaneous microvasculature, thus causing the observed necrotic lesions on the skin. The patient's activated protein $\mathrm{C}$ resistance might have also played a pivotal role by not only lack of its anticoagulatory functions but through its cell adhesion and cytokine modulation effects, resulting in the up-regulation of IL-6 in endothelial cells (71). Previous studies have also reported an increase in serum IL-6 levels following TCZ administration, noting major differences between individual patients (72). The increase in serum IL-6 levels or a TCZ-induced immunogen response may have been the tipping point in precipitating this adverse effect by unbalancing the equilibrium between the coagulatory and anticoagulatory system, resulting in the initiation of immunothrombosis in the cutaneous microvasculature. This process may have also accounted for the significant effectiveness of fresh frozen plasma infusions and the changes in serum D-dimer levels throughout the treatment.

Additionally, it has also been previously shown that IL-6 down-regulates cytochrome P450 (CYP) isozymes (73). As acenocoumarol is metabolized by CYP2C9, blockage of IL6 signaling may therefore result in an altered bioavailability of the medication (74). Notably, no clinical studies investigating the interaction between TCZ and anticoagulants are available, however TCZ-associated thrombotic events have been reported in the medical 
literature $(75,76)$. The concurrent subcutaneous administration of TCZ could have also caused an ineffectiveness of the acenocoumarol therapy, resulting in a procoagulative state, owing to the patient's factor V Leiden heterozygosity. A clinical study by Burmester et al. has also shown that the mean TCZ concentrations in patients receiving subcutaneous injections were twice as high compared to those receiving it intravenously, which would further support this hypothesis and explain why this adverse event did not occur in our patient while she was receiving intravenous TCZ therapy (77).

In closing perspective, this single case could further raise many questions whether the event was an inflammation or thrombosis driven process, also highlighting the additional potential factors of consideration, owing to the clinical complexity deriving from the patient's comorbidities and concurrent medications. As previously mentioned, the clinical experience should compose a crucial factor in addition to the histopathological examination throughout the frameworks of the diagnostic workup. The significance of recognizing the presence of both vasculitis and vasculopathy is underlined by the good therapeutic effect of the employed treatment modalities in our described case(s). Of point, even in situations where the dominant feature of the clinical presentation is inflammation, procoagulative aspects need to be taken into consideration as part of the thrombotic continuum enveloping both hemostasis and immunothrombosis. Subsequent future work will be needed to expand on the dysregulations of the intravascular innate immune responses in specific diseases and to identify underlying triggers for immunothrombosis. Discerning such facilitators will not only expand our understanding of this pathogenesis but will consequently enable the establishment of new diagnostic and therapeutic modalities. 


\section{CONCLUSIONS}

I. Our results suggest that NLR is a potential prognostic marker for systemic involvement in adult IgAV and can be used to identify patients at risk of developing extracutaneous manifestations.

II. We also found that increased pretreatment NLR correlated with the severity of the systemic involvement, which may aid in the stratification of patients into risk groups.

III. In addition to vasculitis, the vasculopathic aspects of CPAN should be recognized as well, giving a considerable role for anticoagulative therapy. Though histopathological assessment regards CPAN as a vasculitis, clinical experience suggests a more complex underlying pathomechanism, where vasculitis and vasculopathy may be intertwined throughout a dynamic clinical evolution.

IV. Further prospective studies on mapping the disease stages of focal vascular occlusion, with CPAN in its focus, should broaden the horizon and help us elucidate the full scope of the underlying pathomechanism. 


\section{ACKNOWLEDGEMENTS}

I would like to express my deepest gratitude to my supervisor and mentor, Professor Zsuzsanna Bata Csörgö, for her continuous support and supervision of my scientific work. She consistently provided valuable guidance and also granted me enough independence during my research and clinical work.

I would like to thank Professor Lajos Kemény for the opportunity to be able to join and conduct my studies at the Department of Dermatology and Allergology of Szeged. Thank you for your valuable advices, management, and for providing a secure background in my scientific works.

I wish to express my special gratitude to my family, for their patience, constant support and encouragement.

Last but not least, I would like thank my colleagues at the Department of Dermatology and Allergology of Szeged and its employees for supporting my scientific work throughout my career. 


\section{REFERENCES}

1. Sunderkötter CH, Zelger B, Chen K-R, Requena L, Piette W, Carlson JA, et al. Nomenclature of Cutaneous Vasculitis: Dermatologic Addendum to the 2012 Revised International Chapel Hill Consensus Conference Nomenclature of Vasculitides. Arthritis Rheumatol (Hoboken, NJ) [Internet] 2018;70:171-84. Available from: http://www.ncbi.nlm.nih.gov/pubmed/29136340

2. Carlson JA, Chen K-R. Cutaneous vasculitis update: small vessel neutrophilic vasculitis syndromes. Am J Dermatopathol [Internet] 2006;28:486-506. Available from: http://www.ncbi.nlm.nih.gov/pubmed/17122493

3. Carlson JA, Ng BT, Chen K-R. Cutaneous vasculitis update: diagnostic criteria, classification, epidemiology, etiology, pathogenesis, evaluation and prognosis. Am J Dermatopathol [Internet] 2005;27:504-28. Available from: http://www.ncbi.nlm.nih.gov/pubmed/16314707

4. Jennette JC, Falk RJ, Bacon PA, Basu N, Cid MC, Ferrario F, et al. 2012 Revised International Chapel Hill Consensus Conference Nomenclature of Vasculitides. Arthritis Rheum [Internet] 2013;65:1-11. Available from: http://doi.wiley.com/10.1002/art.37715

5. Audemard-Verger A, Pillebout E, Guillevin L, Thervet E, Terrier B. IgA vasculitis (Henoch-Shönlein purpura) in adults: Diagnostic and therapeutic aspects. Autoimmun Rev [Internet] Elsevier B.V.; 2015;14:579-85. Available from: http://dx.doi.org/10.1016/j.autrev.2015.02.003

6. Heineke MH, Ballering A V., Jamin A, Ben Mkaddem S, Monteiro RC, Van Egmond M. New insights in the pathogenesis of immunoglobulin A vasculitis (Henoch-Schönlein purpura). Autoimmun Rev [Internet] 2017;16:1246-53. Available from: http://www.ncbi.nlm.nih.gov/pubmed/29037908

7. Davin J-C, Ten Berge IJ, Weening JJ. What is the difference between IgA nephropathy and Henoch-Schönlein purpura nephritis? Kidney Int [Internet] 2001;59:823-34. Available from: https://linkinghub.elsevier.com/retrieve/pii/S0085253815475441

8. Hočevar A, Rotar Z, Ostrovršnik J, Jurčić V, Vizjak A, Dolenc Voljč M, et al. Incidence of IgA vasculitis in the adult Slovenian population. Br J Dermatol 
[Internet] 2014;171:524-7. Available from:

http://www.ncbi.nlm.nih.gov/pubmed/24601900

9. Hočevar A, Rotar Ž, Jurčić V, Čučnik S, Tomšič M. Patient age, gender and extent of purpura may suggest short-term outcomes in adults with IgA vasculitis.

Rheumatology (Oxford) [Internet] 2015;54:1330-2. Available from:

http://www.rheumatology.oxfordjournals.org/lookup/doi/10.1093/rheumatology/kev 122

10. Pillebout E, Thervet E, Hill G, Alberti C, Vanhille P, Nochy D. Henoch-Schönlein Purpura in adults: outcome and prognostic factors. J Am Soc Nephrol [Internet] 2002;13:1271-8. Available from: http://www.ncbi.nlm.nih.gov/pubmed/11961015

11. Makay B, Gücenmez ÖA, Duman M, Ünsal E. The relationship of neutrophil-tolymphocyte ratio with gastrointestinal bleeding in Henoch-Schonlein purpura.

Rheumatol Int [Internet] 2014;34:1323-7. Available from:

http://www.ncbi.nlm.nih.gov/pubmed/24647793

12. Johnson EF, Lehman JS, Wetter DA, Lohse CM, Tollefson MM, E.F. J, et al. Henoch-Schonlein purpura and systemic disease in children: retrospective study of clinical findings, histopathology and direct immunofluorescence in 34 paediatric patients. Br J Dermatol [Internet] 2015;172:1358-63. Available from: www.blackwellpublishing.com/journals/BJD\%5Cnhttp://ovidsp.ovid.com/ovidweb. cgi?T=JS\&PAGE=reference $\& D=$ emed $13 \& N E W S=N \& A N=2015033695 \% 5$ Cnhttp: //ovidsp.ovid.com/ovidweb.cgi?T=JS\&PAGE=reference $\& D=$ prem $\& N E W S=N \& A N$ $=25308217$

13. Nagamori T, Oka H, Koyano S, Takahashi H, Oki J, Sato Y, et al. Construction of a scoring system for predicting the risk of severe gastrointestinal involvement in Henoch-Schönlein Purpura. Springerplus [Internet] 2014;3:171. Available from: http://www.pubmedcentral.nih.gov/articlerender.fcgi?artid=4006069\&tool=pmcentr ez\&rendertype $=$ abstract

14. Zhang L, Wang R, Chen W, Xu X, Dong S, Fan H, et al. Prognostic significance of neutrophil to lymphocyte ratio in patients with gallbladder carcinoma. HPB (Oxford) [Internet] 2016;18:600-7. Available from: http://www.ncbi.nlm.nih.gov/pubmed/27346141 
15. Stotz M, Gerger a, Eisner F, Szkandera J, Loibner H, Ress a L, et al. Increased neutrophil-lymphocyte ratio is a poor prognostic factor in patients with primary operable and inoperable pancreatic cancer. Br J Cancer [Internet] Nature Publishing Group; 2013;109:416-21. Available from: http://www.ncbi.nlm.nih.gov/pubmed/23799847

16. Tanoglu A, Karagoz E. Predictive role of the neutrophil-to-lymphocyte ratio in patients with advanced hepatocellular carcinoma receiving sorafenib. Asian Pac J Cancer Prev [Internet] 2014;15:1063. Available from: http://www.ncbi.nlm.nih.gov/pubmed/24568452

17. Tamhane UU, Aneja S, Montgomery D, Rogers E-K, Eagle KA, Gurm HS. Association between admission neutrophil to lymphocyte ratio and outcomes in patients with acute coronary syndrome. Am J Cardiol [Internet] 2008;102:653-7. Available from: http://www.ncbi.nlm.nih.gov/pubmed/18773982

18. Kwon JH, Jang JW, Kim YW, Lee SW, Nam SW, Jaegal D, et al. The usefulness of $\mathrm{C}$-reactive protein and neutrophil-to-lymphocyte ratio for predicting the outcome in hospitalized patients with liver cirrhosis. BMC Gastroenterol [Internet] 2015;15:146. Available from: http://www.ncbi.nlm.nih.gov/pubmed/26498833

19. Tomasini C. Septic vasculitis and vasculopathy in some infectious emergencies: the perspective of the histopathologist. G Ital Dermatol Venereol [Internet] 2015;150:73-85. Available from: http://www.ncbi.nlm.nih.gov/pubmed/25592669

20. Jenkins J, Babu K, Hsu-Hung E, Robinson-Bostom L, Kroumpouzos G. ANCApositive necrotizing vasculitis and thrombotic vasculopathy induced by levamisoleadulterated cocaine: A distinctive clinicopathologic presentation. J Am Acad Dermatol 2011;65:14-6.

21. Kerk N, Goerge T. Livedoid vasculopathy - a thrombotic disease. Vasa [Internet] 2013;42:317-22. Available from: http://www.ncbi.nlm.nih.gov/pubmed/23989066

22. Papi M, Didona B, De Pità O, Frezzolini A, Di Giulio S, De Matteis W, et al. Livedo vasculopathy vs small vessel cutaneous vasculitis: cytokine and platelet Pselectin studies. Arch Dermatol [Internet] 1998;134:447-52. Available from: http://www.ncbi.nlm.nih.gov/pubmed/9554296

23. Freitas TQ, Halpern I, Criado PR. Livedoid vasculopathy: a compelling diagnosis. 
Autops Case Reports [Internet] 2018;8. Available from:

http://autopsyandcasereports.org/article/doi/10.4322/acr.2018.034

24. Toth C, Trotter M, Clark A, Zochodne D. Mononeuropathy multiplex in association with livedoid vasculitis. Muscle Nerve [Internet] 2003;28:634-9. Available from: http://www.ncbi.nlm.nih.gov/pubmed/14571469

25. Weishaupt C, Strölin A, Kahle B, Kreuter A, Schneider SW, Gerss J, et al. Characteristics, risk factors and treatment reality in livedoid vasculopathy - a multicentre analysis. J Eur Acad Dermatology Venereol [Internet] 2019;jdv.15639. Available from: https://onlinelibrary.wiley.com/doi/abs/10.1111/jdv.15639

26. Criado PR, Rivitti EA, Sotto MN, de Carvalho JF. Livedoid vasculopathy as a coagulation disorder. Autoimmun Rev [Internet] 2011;10:353-60. Available from: https://linkinghub.elsevier.com/retrieve/pii/S1568997210002752

27. De Virgilio A, Greco A, Magliulo G, Gallo A, Ruoppolo G, Conte M, et al. Polyarteritis nodosa: A contemporary overview. Autoimmun Rev [Internet] Elsevier B.V.; 2016;15:564-70. Available from: http://dx.doi.org/10.1016/j.autrev.2016.02.015

28. Alibaz-Oner F, Koster MJ, Crowson CS, Makol A, Ytterberg SR, Salvarani C, et al. Clinical Spectrum of Medium-Sized Vessel Vasculitis. Arthritis Care Res (Hoboken) [Internet] 2017;69:884-91. Available from: http://doi.wiley.com/10.1002/acr.23007

29. Criado PR, Marques GF, Morita TCAB, de Carvalho JF. Epidemiological, clinical and laboratory profiles of cutaneous polyarteritis nodosa patients: Report of 22 cases and literature review. Autoimmun Rev [Internet] Elsevier B.V.; 2016;15:558-63. Available from: http://dx.doi.org/10.1016/j.autrev.2016.02.010

30. Kawakami T, Yamazaki M, Mizoguchi M, Soma Y. High titer of antiphosphatidylserine-prothrombin complex antibodies in patients with cutaneous polyarteritis nodosa. Arthritis Care Res 2007;57:1507-13.

31. Kawakami T, Soma Y. Use of warfarin therapy at a target international normalized ratio of 3.0 for cutaneous polyarteritis nodosa. J Am Acad Dermatol [Internet] Elsevier Inc; 2010;63:602-6. Available from: http://dx.doi.org/10.1016/j.jaad.2009.10.033 
32. Sunderkötter C, Michl C. Hautveränderungen bei Vaskulitiden. Internist (Berl) [Internet] 2019;60:805-13. Available from: http://link.springer.com/10.1007/s00108-019-0642-3

33. Takeuchi S, Soma Y, Kawakami T. IgM in lesional skin of adults with HenochSchönlein purpura is an indication of renal involvement. J Am Acad Dermatol [Internet] 2010;63:1026-9. Available from: http://www.ncbi.nlm.nih.gov/pubmed/20933302

34. Poterucha TJ, Wetter DA, Gibson LE, Camilleri MJ, Lohse CM. Correlates of systemic disease in adult Henoch-Schönlein purpura: a retrospective study of direct immunofluorescence and skin lesion distribution in 87 patients at Mayo Clinic. $\mathbf{J}$ Am Acad Dermatol [Internet] 2012;67:612-6. Available from: http://www.ncbi.nlm.nih.gov/pubmed/22243766

35. Blanco R, Martínez-Taboada VM, Rodríguez-Valverde V, García-Fuentes M, González-Gay MA. Henoch-Schönlein purpura in adulthood and childhood: two different expressions of the same syndrome. Arthritis Rheum [Internet] 1997;40:859-64. Available from: http://www.ncbi.nlm.nih.gov/pubmed/9153547

36. Tancrede-Bohin E, Ochonisky S, Vignon-Pennamen MD, Flageul B, Morel P, Rybojad M. Schönlein-Henoch purpura in adult patients. Predictive factors for IgA glomerulonephritis in a retrospective study of 57 cases. Arch Dermatol [Internet] 1997;133:438-42. Available from: http://www.ncbi.nlm.nih.gov/pubmed/9126006

37. Nam EJ, Kim GW, Kang JW, Im CH, Jeon SW, Cho CM, et al. Gastrointestinal bleeding in adult patients with Henoch-Schonlein purpura. Endoscopy 2014;46:9816.

38. Pillebout E, Thervet E, Hill G, Alberti C, Vanhille P, Nochy D. Henoch-Schönlein Purpura in adults: outcome and prognostic factors. J Am Soc Nephrol [Internet] 2002;13:1271-8. Available from: http://jasn.asnjournals.org/content/13/5/1271.short

39. Hung SP, Yang YH, Lin YT, Wang LC, Lee JH, Chiang BL. Clinical Manifestations and Outcomes of Henoch-Schönlein Purpura: Comparison between Adults and Children. Pediatr Neonatol [Internet] Taiwan Pediatric Association; 2009;50:162-8. Available from: http://dx.doi.org/10.1016/S1875-9572(09)60056-5

40. Zhang Y, Huang X. Gastrointestinal involvement in Henoch-Schönlein purpura. 
Scand J Gastroenterol [Internet] 2008;43:1038-43. Available from:

http://www.tandfonline.com/doi/full/10.1080/00365520802101861

41. Zahorec R. Ratio of neutrophil to lymphocyte counts--rapid and simple parameter of systemic inflammation and stress in critically ill. Bratisl Lek Listy [Internet] 2001;102:5-14. Available from: http://www.ncbi.nlm.nih.gov/pubmed/11723675

42. de Jager CPC, van Wijk PTL, Mathoera RB, de Jongh-Leuvenink J, van der Poll T, Wever PC. Lymphocytopenia and neutrophil-lymphocyte count ratio predict bacteremia better than conventional infection markers in an emergency care unit. Crit Care [Internet] 2010;14:R192. Available from:

http://www.ncbi.nlm.nih.gov/pubmed/21034463

43. Ilan Y, Naparstek Y. Schonlein-Henoch syndrome in adults and children. Semin Arthritis Rheum [Internet] 1991;21:103-9. Available from:

http://www.embase.com/search/results?subaction=viewrecord\&from=export\&id=L2 1322122\%5Cnhttp://dx.doi.org/10.1016/0049-0172(91)90043-

Y\%5Cnhttp://sfx.library.uu.nl/utrecht?sid=EMBASE\&issn=00490172\&id=doi:10.1 016/0049-0172(91)90043-Y\&atitle=Schonlein-Henoch+syndr

44. Park CH, Han DS, Jeong JY, Eun CS, Yoo K-S, Jeon YC, et al. The Optimal CutOff Value of Neutrophil-to-Lymphocyte Ratio for Predicting Prognosis in Adult Patients with Henoch-Schönlein Purpura. PLoS One [Internet] 2016;11:e0153238. Available from: http://dx.plos.org/10.1371/journal.pone.0153238

45. Calvo-Río V, Loricera J, Ortiz-Sanjuán F, Mata C, Martín L, Alvarez L, et al. Revisiting clinical differences between hypersensitivity vasculitis and HenochSchönlein purpura in adults from a defined population. Clin Exp Rheumatol [Internet] 2014;32:S34-40. Available from: http://www.ncbi.nlm.nih.gov/pubmed/24528895

46. Alalwani M, Billings SD, Gota CE. Clinical significance of immunoglobulin deposition in leukocytoclastic vasculitis: a 5-year retrospective study of 88 patients at cleveland clinic. Am J Dermatopathol [Internet] 2014;36:723-9. Available from: http://www.ncbi.nlm.nih.gov/pubmed/25014103

47. Hočevar A, Rotar Z, Jurčić V, Pižem J, Čučnik S, Vizjak A, et al. IgA vasculitis in adults: the performance of the EULAR/PRINTO/PRES classification criteria in 
adults. Arthritis Res Ther [Internet] 2015;18:58. Available from: http://www.pubmedcentral.nih.gov/articlerender.fcgi?artid=4774143\&tool=pmcentr ez\&rendertype $=$ abstract

48. Gardette E, Moguelet P, Bouaziz J, Lipsker D, Dereure O, Pelletier F, et al. Livedoid Vasculopathy: A French Observational Study Including Therapeutic Options. Acta Derm Venereol [Internet] 2018;98:842-7. Available from: https://www.medicaljournals.se/acta/content/abstract/10.2340/00015555-2965

49. Gibbs MB, English JC, Zirwas MJ. Livedo reticularis: an update. J Am Acad Dermatol [Internet] 2005;52:1009-19. Available from:

http://www.ncbi.nlm.nih.gov/pubmed/15928620

50. Criado PR, Marques GF, Morita TCAB, de Carvalho JF. Epidemiological, clinical and laboratory profiles of cutaneous polyarteritis nodosa patients: Report of 22 cases and literature review. Autoimmun Rev [Internet] 2016;15:558-63. Available from: http://www.ncbi.nlm.nih.gov/pubmed/26876385

51. Llamas-Velasco M, de Argila D, Fraga J, García-Diez A. Cutaneous Polyarteritis Nodosa With Manifestations of Livedoid Vasculopathy. Actas DermoSifiliográficas (English Ed [Internet] 2011;102:477-9. Available from: https://linkinghub.elsevier.com/retrieve/pii/S1578219011000114

52. Toth C, Trotter M, Clark A, Zochodne D. Mononeuropathy multiplex in association with livedoid vasculitis. Muscle Nerve [Internet] 2003;28:634-9. Available from: http://doi.wiley.com/10.1002/mus.10450

53. Llamas-Velasco M, Alegría V, Santos-Briz Á, Cerroni L, Kutzner H, Requena L. Occlusive Nonvasculitic Vasculopathy. Am J Dermatopathol [Internet] 2017;39:637-62. Available from: http://www.ncbi.nlm.nih.gov/pubmed/27759698

54. Hutachuda P, Hanamornroongruang S, Pattanaprichakul P, Chanyachailert P, Sitthinamsuwan P. Interobserver reliability of histopathological features for distinguishing between cutaneous polyarteritis nodosa and superficial thrombophlebitis. Histopathology [Internet] 2018;73:407-16. Available from: http://www.ncbi.nlm.nih.gov/pubmed/29675878

55. Khenifer S, Thomas L, Balme B, Dalle S. Livedoid vasculopathy: thrombotic or inflammatory disease? Clin Exp Dermatol [Internet] 2010;35:693-8. Available from: 
http://doi.wiley.com/10.1111/j.1365-2230.2009.03732.x

56. Mimouni D, Ng PP, Rencic A, Nikolskaia OV, Bernstein BD, Nousari HC.

Cutaneous polyarteritis nodosa in patients presenting with atrophie blanche. $\mathrm{Br} \mathrm{J}$

Dermatol [Internet] 2003;148:789-94. Available from:

http://doi.wiley.com/10.1046/j.1365-2133.2003.05176.x

57. Engelmann B, Massberg S. Thrombosis as an intravascular effector of innate immunity. Nat Rev Immunol [Internet] 2013;13:34-45. Available from: http://www.ncbi.nlm.nih.gov/pubmed/23222502

58. Zinsser HH, Pryde AW. Experimental study of physical factors, including fibrin formation, influencing the spread of fluids and small particles within and from the peritoneal cavity of the dog. Ann Surg [Internet] 1952;136:818-27. Available from: http://journals.lww.com/00000658-195211000-00006

59. Müller I, Klocke A, Alex M, Kotzsch M, Luther T, Morgenstern E, et al. Intravascular tissue factor initiates coagulation via circulating microvesicles and platelets. FASEB J [Internet] 2003;17:476-8. Available from: http://www.ncbi.nlm.nih.gov/pubmed/12514112

60. Fuchs TA, Brill A, Duerschmied D, Schatzberg D, Monestier M, Myers DD, et al. Extracellular DNA traps promote thrombosis. Proc Natl Acad Sci U S A [Internet] 2010;107:15880-5. Available from: http://www.ncbi.nlm.nih.gov/pubmed/20798043

61. Kambas K, Chrysanthopoulou A, Vassilopoulos D, Apostolidou E, Skendros P, Girod A, et al. Tissue factor expression in neutrophil extracellular traps and neutrophil derived microparticles in antineutrophil cytoplasmic antibody associated vasculitis may promote thromboinflammation and the thrombophilic state associated with the disease. Ann Rheum Dis [Internet] 2014;73:1854-63. Available from: http://www.ncbi.nlm.nih.gov/pubmed/23873874

62. Kessenbrock K, Krumbholz M, Schönermarck U, Back W, Gross WL, Werb Z, et al. Netting neutrophils in autoimmune small-vessel vasculitis. Nat Med [Internet] 2009;15:623-5. Available from: http://www.ncbi.nlm.nih.gov/pubmed/19448636

63. Knight JS, Carmona-Rivera C, Kaplan MJ. Proteins derived from neutrophil extracellular traps may serve as self-antigens and mediate organ damage in autoimmune diseases. Front Immunol [Internet] 2012;3:380. Available from: 
http://www.ncbi.nlm.nih.gov/pubmed/23248629

64. Emmi G, Silvestri E, Squatrito D, Amedei A, Niccolai E, D’Elios MM, et al. Thrombosis in vasculitis: from pathogenesis to treatment. Thromb J [Internet] 2015;13:15. Available from: http://www.ncbi.nlm.nih.gov/pubmed/25883536

65. Guillevin L, Mouthon L. Tumor necrosis factor-alpha blockade and the risk of vasculitis. J Rheumatol [Internet] 2004;31:1885-7. Available from: http://www.ncbi.nlm.nih.gov/pubmed/15468348

66. Sato K, Tsuchiya M, Saldanha J, Koishihara Y, Ohsugi Y, Kishimoto T, et al. Reshaping a human antibody to inhibit the interleukin 6-dependent tumor cell growth. Cancer Res [Internet] 1993;53:851-6. Available from: http://www.ncbi.nlm.nih.gov/pubmed/8428365

67. Matsuo Y, Mizoguchi F, Kohsaka H, Ito E, Eishi Y, Miyasaka N. Tocilizumabinduced immune complex glomerulonephritis in a patient with rheumatoid arthritis. Rheumatology (Oxford) [Internet] 2013;52:1341-3. Available from: http://www.ncbi.nlm.nih.gov/pubmed/23325036

68. Sakaue S, Sumitomo S, Kubo K, Fujio K, Yamamoto K, Keishi F, et al. Tocilizumab-induced leucocytoclastic vasculitis in a patient with rheumatoid arthritis. Rheumatology (Oxford) [Internet] 2014;53:1529-30. Available from: http://www.ncbi.nlm.nih.gov/pubmed/24609062

69. Stouthard JM, Levi M, Hack CE, Veenhof CH, Romijn HA, Sauerwein HP, et al. Interleukin-6 stimulates coagulation, not fibrinolysis, in humans. Thromb Haemost [Internet] 1996;76:738-42. Available from: http://www.ncbi.nlm.nih.gov/pubmed/8950783

70. Kerr R, Stirling D, Ludlam CA. Interleukin 6 and Haemostasis. Br J Haematol [Internet] 2001;115:3-12. Available from: http://doi.wiley.com/10.1046/j.13652141.2001.03061.x

71. Hooper WC, Phillips DJ, Renshaw MA, Evatt BL, Benson JM. The up-regulation of IL-6 and IL-8 in human endothelial cells by activated protein C. J Immunol [Internet] 1998;161:2567-73. Available from: http://www.ncbi.nlm.nih.gov/pubmed/9725257 
72. Nishimoto N, Terao K, Mima T, Nakahara H, Takagi N, Kakehi T. Mechanisms and pathologic significances in increase in serum interleukin-6 (IL-6) and soluble IL-6 receptor after administration of an anti-IL-6 receptor antibody, tocilizumab, in patients with rheumatoid arthritis and Castleman disease. Blood 2008;112:3959-64.

73. Abdel-Razzak Z, Loyer P, Fautrel A, Gautier JC, Corcos L, Turlin B, et al. Cytokines down-regulate expression of major cytochrome P-450 enzymes in adult human hepatocytes in primary culture. Mol Pharmacol [Internet] 1993;44:707-15. Available from: http://www.ncbi.nlm.nih.gov/pubmed/8232220

74. Thijssen HH, Flinois JP, Beaune PH. Cytochrome P4502C9 is the principal catalyst of racemic acenocoumarol hydroxylation reactions in human liver microsomes. Drug Metab Dispos [Internet] 2000;28:1284-90. Available from: http://www.ncbi.nlm.nih.gov/pubmed/11038154

75. Clarivet B, Robin P, Pers YM, Ferreira R, Lebrun J, Jorgensen C, et al. Tocilizumab and mesenteric arterial thrombosis: drug-drug interaction with anticoagulants metabolized by CYP 450 and/or by P-glycoprotein. Eur J Clin Pharmacol [Internet] European Journal of Clinical Pharmacology; 2016;72:1413-4. Available from: http://link.springer.com/10.1007/s00228-016-2107-0

76. Jewell P, Ansorge O, Kuker W, Irani SR, Zamboni G. Tocilizumab-associated multifocal cerebral thrombotic microangiopathy. Neurol Clin Pract 2016;6:e24-6.

77. Burmester GR, Rubbert-Roth A, Cantagrel A, Hall S, Leszczynski P, Feldman D, et al. Efficacy and safety of subcutaneous tocilizumab versus intravenous tocilizumab in combination with traditional DMARDs in patients with RA at week 97 (SUMMACTA). Ann Rheum Dis [Internet] 2015;1-7. Available from: http://ard.bmj.com/cgi/doi/10.1136/annrheumdis-2015-207281 
I. 


\title{
Neutrophil-to-lymphocyte ratio: a biomarker for predicting systemic involvement in adult IgA vasculitis patients
}

\author{
G.R. Nagy, ${ }^{*}$ L. Kemény, Z. Bata-Csörgő \\ Department of Dermatology and Allergology, University of Szeged, Szeged, Hungary \\ ${ }^{*}$ Correspondence: G.R. Nagy. E-mail: n.geza@outlook.com
}

\begin{abstract}
Background IgA vasculitis (IgAV) is a small-vessel leucocytoclastic cutaneous vasculitis, often associated with kidney and gastrointestinal (Gl) manifestations. Although predictive factors for systemic involvement have been extensively studied in children, there is paucity in the literature regarding adult patients. Neutrophil-to-lymphocyte ratio (NLR) is an inflammatory marker, used to assess systemic inflammation in various diseases.

Objective We sought to evaluate whether NLR can be used for predicting renal and Gl involvement in adult IgA vasculitis patients.

Methods This was a retrospective review of adult patients who were diagnosed with IgAV at our institution between 2004 and 2016.

Results A total of 40 patients met our inclusion criteria. Half of the enrolled patients had clinical symptoms suggestive of systemic involvement, of which $6(15 \%)$ had only renal, $3(7.5 \%)$ had only GI and $11(27.5 \%)$ had both renal and GI involvement. Pretreatment NLR was significantly associated with renal and/or GI manifestations of the disease $(P<0.001)$. The optimal cut-off value of NLR, for predicting systemic involvement was 3.34 , with a specificity of $95 \%$ and a sensitivity of $85 \%$. In addition, pretreatment NLR was also found to be significantly correlated with the severity of the systemic manifestations of $\operatorname{lgAV}(P=0.022)$.
\end{abstract}

Conclusion This study suggests that NLR is a potential indicator for prognosticating systemic involvement in adult IgAV.

Received: 26 September 2016; Accepted: 6 February 2017

\section{Conflict of Interest Disclosure}

None Declared.

\section{Funding sources}

None.

\section{Introduction}

Immunoglobulin A vasculitis (IgAV), formerly known as Henoch-Schönlein purpura, is an immune complex-mediated small-vessel leucocytoclastic cutaneous vasculitis, characterized by palpable purpura, arthralgia or arthritis, gastrointestinal (GI) and renal involvement. ${ }^{1}$ It is often regarded as a disease of childhood, but contrary to popular belief, it is not uncommon in adults. $^{2}$ Although it is considered to be the same entity, the clinical manifestations and disease course differ greatly in these two age groups. Previous studies have demonstrated that unlike in children, adult patients develop systemic involvement more frequently, with a high risk of severe GI bleeding and chronic kidney disease. ${ }^{3,4}$ This highlights the importance and the need of prognostic markers that can help identify IgAV patients who are at risk of developing unfavourable extracutaneous manifestations. While predictive factors have been extensively studied in children, there are limited data on adults. ${ }^{5-7}$
Blood neutrophil-to-lymphocyte ratio (NLR) is an inexpensive and easily obtainable laboratory marker for quantifying systemic inflammation, which has been used to predict clinical outcomes in patients with various internal malignancies, cardiovascular disease and liver cirrhosis. ${ }^{8-12}$ As this ratio integrates information on two immune pathways, it may provide a predictive ability that outweighs other inflammatory parameters. The aim of this study was to evaluate the utility of this ratio in predicting renal and GI involvement in adult IgAV patients.

\section{Patients and methods}

\section{Patients}

A retrospective review of adult patients diagnosed with IgAV between January 2004 and January 2016 was performed. In accordance with the study criteria used by Takeuchi et al. ${ }^{13}$ and Poterucha et al., ${ }^{14}$ patients needed to have palpable purpura 
consistent with the disease, skin biopsy specimen showing leucocytoclastic vasculitis on light microscopy and IgA deposition on direct immunofluorescence.

Patients were excluded if they had an immunologic comorbidity, coexisting internal malignancy, haematological disorder, cryoglobulinemia or any chronic renal or GI diseases. Additionally, patients who experienced hematochezia, melena or hematemesis 2 days before or after blood sampling, were also excluded owing to the possibility of neutrophilia being the secondary effect of an acute haemorrhage.

The study was approved by the local ethics review committee.

\section{Data collection}

We analyzed the medical records and registered the following: gender, age, duration of symptoms before blood sampling, clinical symptoms, results of laboratory testing and initial treatment. The laboratory test results included C-reactive protein (CRP), erythrocyte sedimentation rate (ESR), white blood cell count (WBC), neutrophil and lymphocyte counts, haematological parameters, urine and stool examinations. NLR was calculated based on the initial complete blood count test, by dividing the neutrophil count with the lymphocyte count. By reviewing the ambulatory records, we also registered any subsequent renal or GI symptoms following initial remission. Patients were followed from baseline visit until loss of follow-up or death.

\section{Assessment of gastrointestinal and renal involvement}

We defined GI involvement as clinical signs of hematochezia, melena or hematemesis; or a positive test result for faecal haemoglobin. Renal involvement was determined through haematuria ( $>5$ red blood cells per high-power microscopic field in a centrifuged specimen; in the absence of concurrent urinary tract infections, urolithiasis and anticoagulant therapy) or proteinuria $(>150 \mathrm{mg} / 24 \mathrm{~h})$, or presence of predominant mesangial IgA deposition on the renal biopsy specimen.

\section{Statistical analysis}

The normality of distribution was analyzed using the ShapiroWilk test and parametric or non-parametric statistical tests were used accordingly. Quantitative variables are displayed as mean \pm standard deviation (SD) or with median and interquartile range (IQR). Continuous variables were compared using one-way analysis of variance or Kruskal-Wallis test for multiple groups and independent sample $t$-test or Mann-Whitney $U$ test for dual groups. Categorical features are summarized with frequency count, percentage and were compared using Fisher's exact test. The intercorrelations between parameters were examined using Spearman's rank correlation coefficient test. A receiver operating characteristic (ROC) curve was performed to examine the prognostic utility of NLR and to identify the optimal cut-off value. Statistical analyses were performed using the Statistical Package for Social Science version 22 (SPSS Inc., Chicago, IL, USA) for Windows. All tests were two-tailed and $P$-values of less than 0.05 were considered statistically significant.

\section{Results}

\section{Basic characteristics of the study sample}

Forty adult patients with IgAV who met the inclusion criteria were identified. The median age was 61 years (range 1982 years). The cohort included $23(57.5 \%)$ females and 17 $(42.5 \%)$ males. Half of the patients had clinical symptoms suggestive of GI and/or renal involvement, of which six (15\%) had only renal, three $(7.5 \%)$ had only GI and 11 (27.5\%) had both renal and GI involvement. Because the presence or absence of arthritis and arthralgia was only recorded in 23 (57.5\%) patients of the enrolled 40 cases, we have not included this data in the statistical analysis. The median time between the appearance of cutaneous symptoms and initial blood analysis was 9.5 days (IQR 5-14 days). Patients received initial treatment after blood sampling and in all cases either corticosteroid monotherapy or a combination of corticosteroid and antibiotics were employed. Based on the severity of the subsequently developed renal and GI symptoms, therapies were altered accordingly at the discretion of the clinician.

\section{Clinical features}

As NLR represents an inflammatory response, we also included other routinely used inflammatory markers for comparison. Spearman correlation analysis indicated a significant correlation of NLR with CRP $(\rho=0.482 ; P=0.002)$, ESR $(\rho=0.37$; $P=0.019)$ and WBC $(\rho=0.469 ; P=0.002)$; however, no significant correlation was observed with the duration of symptoms before blood sampling $(\rho=-0.269 ; P=0.094)$ or patient age ( $\rho=0.282 ; P=0.078$ ). To further exclude the possibility of an acute haemorrhage having an effect on NLR, we compared the haematological laboratory values of the enrolled patients based on their organ involvements, which did not indicate a significant difference between the groups (Table 1).

When stratifying patients based on their renal and GI manifestations and comparing the inflammatory laboratory parameters, there were no statistically significant differences, irrespective of which organ involvement the patients had (Table 2). Intriguingly, CRP was quite low in those with only GI involvement, which may be due to the small number of patients in this group and their mild clinical manifestations of IgAV. These three patients did not have extensive cutaneous symptoms and only displayed faecal haemoglobin positivity without any macroscopic bleeding of the GI tract.

Because there were no statistically significant differences in the haematological and the inflammatory values following patient stratification with regard to their organ involvements, the enrolled cases were divided into two groups. 
Table 1 Haematological laboratory values of enrolled patients based on their organ involvements

\begin{tabular}{|c|c|c|c|c|c|}
\hline Variable & $\begin{array}{l}\text { Renal involvement } \\
(n=6)\end{array}$ & $\begin{array}{l}\text { GI involvement } \\
(n=3)\end{array}$ & $\begin{array}{l}\text { Renal and GI } \\
\text { involvement }(n=11)\end{array}$ & $\begin{array}{l}\text { No renal or GI } \\
\text { involvement }(n=20)\end{array}$ & $P$-value* \\
\hline Haemoglobin ( $\mathrm{g} / \mathrm{dL})$, mean $\pm \mathrm{SD}$ & $128.5 \pm 26.8$ & $144.7 \pm 19.6$ & $127.6 \pm 20.3$ & $133 \pm 19$ & 0.483 \\
\hline Haematocrit $(L / L)$, mean $\pm S D$ & $0.38 \pm 0.1$ & $0.42 \pm 0.1$ & $0.38 \pm 0.1$ & $0.39 \pm 0.1$ & 0.628 \\
\hline Erythrocyte count $\left(\times 10^{12} / L\right)$, mean $\pm S D$ & $4.39 \pm 0.9$ & $4.74 \pm 0.49$ & $4.26 \pm 0.49$ & $4.45 \pm 0.53$ & 0.591 \\
\hline Platelet count $\left(\times 10^{9} / \mathrm{L}\right)$, mean $\pm \mathrm{SD}$ & $278.5 \pm 61.9$ & $240.7 \pm 20.9$ & $306.7 \pm 132.8$ & $282.1 \pm 71.9$ & 0.558 \\
\hline
\end{tabular}

${ }^{*}$ Continuous variables were compared using the Kruskal-Wallis test.

Table 2 Inflammatory laboratory values of patients with systemic manifestations based on their organ involvements

\begin{tabular}{lllc} 
Variable & $\begin{array}{l}\text { Renal involvement } \\
(\boldsymbol{n}=\mathbf{6})\end{array}$ & $\begin{array}{l}\text { Gl involvement } \\
(\boldsymbol{n}=\mathbf{3})\end{array}$ & \multicolumn{1}{c}{$\begin{array}{l}\boldsymbol{P} \text {-value* } \\
(\boldsymbol{n}=\mathbf{1 1})\end{array}$} \\
\hline CRP $(\mathrm{mg} / \mathrm{L})$, mean \pm SD & $85.3 \pm 48.2$ & $13 \pm 12.3$ & $105 \pm 93.3$ \\
ESR $(\mathrm{mm} / \mathrm{h})$, mean \pm SD & $59.2 \pm 44.7$ & $24.3 \pm 20.8$ & $50 \pm 28.8$ \\
WBC $\left(\times 10^{9} / \mathrm{L}\right)$, mean \pm SD & $9.3 \pm 2.5$ & $11 \pm 2.9$ & $12.1 \pm 5.4$ \\
NLR, mean \pm SD & $4.2 \pm 1.7$ & $5.4 \pm 1.5$ & 0.334 \\
\hline
\end{tabular}

CRP, C-reactive protein; ESR, erythrocyte sedimentation rate; GI, gastrointestinal; NLR, neutrophil-to-lymphocyte rate; WBC, white blood cell count. ${ }^{*}$ Continuous variables were compared using the Kruskal-Wallis test.

While group 1 included patients who only had cutaneous symptoms, group 2 consisted of patients who developed GI and/or renal manifestations of IgAV, in addition to the cutaneous symptoms. The demographic and clinical characteristics of these two groups are detailed in Table 3. Of the registered inflammatory markers, CRP $(P=0.002)$ and NLR $(P<0.001)$ were significantly higher in group 2 , whereas the other laboratory parameters, age, gender and the duration of symptoms were not statistically associated with systemic involvement.

Six (30\%) patients in group 2 displayed GI involvement with the presence of macroscopic bleeding, whereas eight (40\%) only had faecal haemoglobin positivity without a clinically apparent haemorrhage. In cases where gross blood was observed, an endoscopic examination was performed, which showed IgAV compatible macroscopic image in all of these patients.

With regard to renal symptoms, proteinuria was a frequent finding among those with a systemic involvement ( $n=15 ; 75 \%)$; however, none of the patients developed nephrotic syndrome. In two patients, renal involvement progressed into end-stage renal disease, 11 and 14 days following initial blood analysis. A kidney biopsy was performed in both cases, which confirmed the association with IgAV. Additionally, these two individuals also developed severe GI bleeding, the former with intussusception and the latter with perforation as a secondary complication, both requiring surgical intervention.

\section{Receiver operating characteristic curves of NLR versus other inflammatory markers}

ROC curves of NLR and other inflammatory markers in relation to the systemic involvement are depicted in Fig. 1. The area under the curve (AUC) for NLR, CRP, ESR and WBC was 0.892
Table 3 Demographics and clinical findings of patients with respect to their systemic involvement

\begin{tabular}{|c|c|c|c|}
\hline Variable & $\begin{array}{l}\text { Group } 1 \\
(n=20)\end{array}$ & $\begin{array}{l}\text { Group } 2 \\
(n=20)\end{array}$ & $P$-value* \\
\hline $\begin{array}{l}\text { Age; years, } \\
\text { mean } \pm \text { SD }\end{array}$ & $56 \pm 20$ & $60 \pm 16.3$ & 0.493 \\
\hline Gender, $n(\%)$ & & & 0.056 \\
\hline Female & $13(65)$ & $6(30)$ & NA \\
\hline Male & $7(35)$ & $14(70)$ & NA \\
\hline $\begin{array}{l}\text { Duration of symptoms; } \\
\text { days, median (IQR) }\end{array}$ & $11(7-15.3)$ & $7.5(4-12.5)$ & 0.081 \\
\hline $\begin{array}{l}\mathrm{CRP}(\mathrm{mg} / \mathrm{L}) \\
\text { median (IQR) }\end{array}$ & $13.1(7.3-48.9)$ & $68.2(25.7-124.3)$ & 0.002 \\
\hline $\begin{array}{l}\mathrm{ESR}(\mathrm{mm} / \mathrm{h}) \\
\text { median (IQR) }\end{array}$ & $22(10-34.3)$ & $43.5(21.5-79.3)$ & 0.068 \\
\hline $\begin{array}{l}\text { WBC }\left(\times 10^{9} / \mathrm{L}\right) \\
\text { median }(\mathrm{IQR})\end{array}$ & $8.2(6.6-12)$ & $10.9(8.1-12.3)$ & 0.142 \\
\hline NLR, median (IQR) & $2.6(1.6-3)$ & $5.9(4.3-7.1)$ & $<0.001$ \\
\hline $\begin{array}{l}\text { Follow-up; months, } \\
\text { median (IQR) }\end{array}$ & $6.8(3.5-24.9)$ & $5.3(2.1-19.1)$ & NA \\
\hline $\begin{array}{l}\text { End-stage renal } \\
\text { disease, } n(\%)\end{array}$ & $0(0)$ & $2(10)$ & NA \\
\hline Death, $n(\%)$ & $0(0)$ & $1(5)$ & NA \\
\hline
\end{tabular}

CRP, C-reactive protein; ESR, erythrocyte sedimentation rate; IQR, interquartile range; NLR, neutrophil-to-lymphocyte rate; WBC, white blood cell count.

*Fisher's exact test was used to compare categorical variables. Student's ttest was used to compare age and Mann-Whitney $U$ test was used to compare the duration of symptoms and laboratory values.

(95\% CI: $0.785-1 ; P<0.001), 0.779$ (95\% CI: $0.635-0.922$; $P=0.003$ ), 0.669 (95\% CI: $0.498-0.839 ; P=0.068)$ and 0.637 (95\% CI $0.462-0.813 ; P=0.089$ ), respectively. Of the considered laboratory data, NLR provided the strongest diagnostic value, as indicated by the highest AUC value. The optimal cut-off value of 


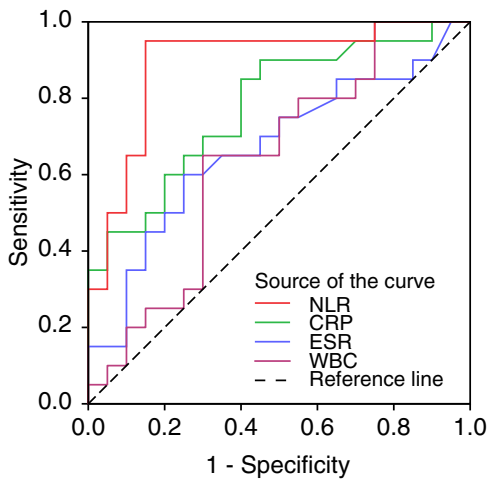

Figure 1 Receiver operating characteristic curves for predicting the development of renal and/or gastrointestinal involvement. CRP, C-reactive protein; ESR, erythrocyte sedimentation rate; NLR, neutrophil-to-lymphocyte ratio; WBC, white blood cell count.

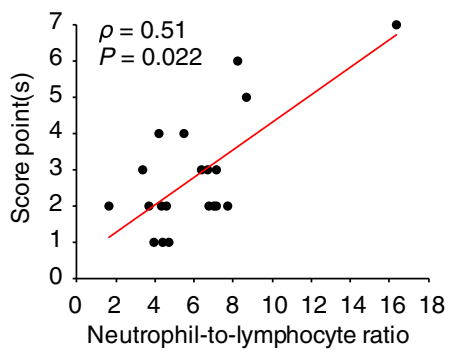

Figure 2 Correlation between pretreatment neutrophil-to-lymphocyte ratio and disease severity score. The Spearman correlation test was used, giving the coefficient $\rho$, with $P$-value $<0.05$ being significant.

NLR for predicting systemic involvement was 3.34, with a specificity of $95 \%$ and a sensitivity of $85 \%$.

\section{NLR and disease severity}

We further looked at the correlation of NLR and disease severity in patients with renal and/or GI involvement. For this, we have constructed a simple 7-point scoring system based on the clinical manifestations and course of the disease, observed among our enrolled cases. Patients received 1 point for each of the following features: haematuria, proteinuria, renal impairment, faecal haemoglobin positivity, macroscopic bleeding from the GI tract, the necessity of intensive care/surgery/dialysis or blood transfusion and death. NLR was found to be significantly correlated with the disease severity score $(\rho=0.51 ; P=0.022)$ (Fig. 2).

\section{Discussion}

Although IgAV is often a self-limiting and benign disease in children, severe complications may occur in adults, including renal impairment and serious GI bleeding requiring intensive care or surgery. ${ }^{3,15}$ A number of studies have been conducted with the aim to correlate various clinical features with systemic involvement in adults, such as direct immunofluorescence findings of skin biopsy specimens and skin lesion distribution, although the results are dissenting. ${ }^{13,14,16}$ Additionally, endoscopic features of GI lesions and histopathological findings of renal biopsies have also been studied; however, these modalities can cause serious complications and may be contraindicated in certain cases. ${ }^{4,17}$ Therefore, to gain more prognostic information, we examined the utility of a noninvasive and easily obtainable cost-effective laboratory parameter. To our knowledge, this is the first biopsyproven case-control study to investigate the predictive value of NLR for systemic involvement in adult IgAV patients.

Half of the patients included in our study developed renal and/or GI involvement, of which the majority concurrently had both organs affected. In accordance with this finding, other studies have also noted high incidence of simultaneous renal and GI manifestations in adult IgAV. ${ }^{4,18}$ It should be mentioned, however, that some researchers could not confirm this finding, which may be related to the varying criteria employed for defining systemic involvement. ${ }^{13,19}$ Male gender has also been previously described as a poor prognostic indicator for systemic involvement; however, our results did not reach statistical significance. $^{3,18}$

When stratifying patients based on their renal and GI involvement and comparing the registered laboratory results, there were no significant differences in the haematological and the inflammatory laboratory values, which implies that NLR and the other inflammatory markers are not organ-specific prognostic indicators and their elevated values were not the secondary effect of a severe bleeding. Consequently, the high NLR observed in our study is, therefore, likely to be the result of an inflammatory response.

Our results demonstrated that of all the considered inflammatory parameters, NLR had the strongest diagnostic value. The optimal cut-off point for predicting systemic involvement was 3.34 with a specificity of $95 \%$ and a sensitivity of $85 \%$. Additionally, we also found that pretreatment NLR values significantly correlated with the severity of the disease in patients who developed systemic involvement. It is important to note, however, that when constructing the scoring system used to evaluate this correlation in our study, we only considered the presence or absence of certain clinical manifestations, rather than assessing the extent of renal or GI symptoms on a spectrum.

Progression to end-stage renal disease was observed in two individuals. Compared to children, adult IgAV patients are more likely to present with a delayed renal involvement, often requiring close monitoring and diligent testing for even up to 6 months following the onset of IgAV, despite favourable initial laboratory results. ${ }^{20}$ While delayed renal involvement was not observed in any of our cases, it should be noted that 20 patients (10 individuals from group 1 and 10 individuals from group 2) 
did not have a follow-up time period of at least 6 months. Although one of the patients with a lower NLR than the cut-off value identified in our study developed renal manifestations of IgAV (microscopic haematuria and proteinuria) during his admission, he remained asymptomatic throughout the followup time period ( 9 years).

More than a third of our patients had GI involvement, which is in accordance with previous reports. ${ }^{3,4}$ In most cases, the symptoms consisted of colicky abdominal pain with faecal haemoglobin positivity; however, some patients developed macroscopic bleeding. Severe complications, such as intussusception and perforation were also observed in two cases, the latter resulting in a fatal outcome. None of the patients in our cohort with a lower NLR value than 3.34 displayed any clinical signs suggestive of GI involvement, during their admission and follow-up, which further highlights the prognostic value of NLR. Although a recent study demonstrated the applicability of this ratio with regard to GI bleeding in adult patients with cutaneous vasculitis, the employed criteria for the diagnosis of IgAV did not include skin histopathology examination. ${ }^{21}$ In consideration of the probable clinical overlap between hypersensitivity vasculitis and IgAV, differentiating between these two entities is imperative when assessing and predicting renal or GI involvement. ${ }^{22}$ Consequently, the clinical significance of the presence or absence of IgA deposition found by direct immunofluorescence of the cutaneous biopsy specimen should not be overlooked, as it enhances the diagnostic specificity. ${ }^{1,23,24}$

The significance of our findings is limited by the retrospective nature of this study and its single-centre sample size. Nevertheless, as our selection was based on both clinical and histopathological findings, with the exclusion of patients with other background diseases, we believe our data represent accurate observations. Larger prospective multi-centre studies are required to validate our findings and to establish a generally acceptable cut-off value of this ratio.

In conclusion, our results suggest that NLR is a potential prognostic marker for systemic involvement in adult IgAV and can be used to identify patients at risk of developing extracutaneous manifestations. In addition, we also found that increased pretreatment NLR correlated with the severity of the systemic involvement. Whether this ratio may permit the design of prospective therapeutic studies remains to be determined.

\section{References}

1 Jennette JC, Falk RJ, Bacon PA et al. 2012 Revised international Chapel Hill consensus conference nomenclature of vasculitides. Arthritis Rheum 2013; 65: 1-11.

2 Hočevar A, Rotar Z, Ostrovršnik J et al. Incidence of IgA vasculitis in the adult Slovenian population. Br J Dermatol 2014; 171: 524-527.

3 Hočevar A, Rotar Ž, Jurčić V et al. Patient age, gender and extent of purpura may suggest short-term outcomes in adults with IgA vasculitis. Rheumatology (Oxford) 2015; 54: 1330-1332.
4 Pillebout E, Thervet E, Hill G et al. Henoch-Schönlein purpura in adults: outcome and prognostic factors. J Am Soc Nephrol 2002; 13: 1271-1278.

5 Makay B, Gücenmez ÖA, Duman M, Ünsal E. The relationship of neutrophil-to-lymphocyte ratio with gastrointestinal bleeding in HenochSchonlein purpura. Rheumatol Int 2014; 34: 1323-1327.

6 Johnson EF, Lehman JS, Wetter DA et al. Henoch-Schonlein purpura and systemic disease in children: retrospective study of clinical findings, histopathology and direct immunofluorescence in 34 paediatric patients. Br J Dermatol 2015; 172: 1358-1363.

7 Nagamori T, Oka H, Koyano S et al. Construction of a scoring system for predicting the risk of severe gastrointestinal involvement in HenochSchönlein purpura. Springerplus 2014; 3: 171.

8 Zhang L, Wang R, Chen W et al. Prognostic significance of neutrophil to lymphocyte ratio in patients with gallbladder carcinoma. HPB (Oxford) 2016; 18: 600-607.

9 Stotz M, Gerger A, Eisner F et al. Increased neutrophil-lymphocyte ratio is a poor prognostic factor in patients with primary operable and inoperable pancreatic cancer. Br J Cancer 2013; 109: 416-421.

10 Tanoglu A, Karagoz E. Predictive role of the neutrophil-to-lymphocyte ratio in patients with advanced hepatocellular carcinoma receiving sorafenib. Asian Pac J Cancer Prev 2014; 15: 1063.

11 Tamhane UU, Aneja S, Montgomery D et al. Association between admission neutrophil to lymphocyte ratio and outcomes in patients with acute coronary syndrome. Am J Cardiol 2008; 102: 653-657.

12 Kwon JH, Jang JW, Kim YW et al. The usefulness of C-reactive protein and neutrophil-to-lymphocyte ratio for predicting the outcome in hospitalized patients with liver cirrhosis. BMC Gastroenterol 2015; 15: 146.

13 Takeuchi S, Soma Y, Kawakami T. IgM in lesional skin of adults with Henoch-Schönlein purpura is an indication of renal involvement. J Am Acad Dermatol 2010; 63: 1026-1029.

14 Poterucha TJ, Wetter DA, Gibson LE et al. Correlates of systemic disease in adult Henoch-Schönlein purpura: a retrospective study of direct immunofluorescence and skin lesion distribution in 87 patients at Mayo Clinic. J Am Acad Dermatol 2012; 67: 612-616.

15 Blanco R, Martínez-Taboada VM, Rodríguez-Valverde V et al. HenochSchönlein purpura in adulthood and childhood: two different expressions of the same syndrome. Arthritis Rheum 1997; 40: 859-864.

16 Tancrede-Bohin E, Ochonisky S, Vignon-Pennamen MD et al. SchönleinHenoch purpura in adult patients. Predictive factors for IgA glomerulonephritis in a retrospective study of 57 cases. Arch Dermatol 1997; 133: 438-442.

17 Nam EJ, Kim GW, Kang JW et al. Gastrointestinal bleeding in adult patients with Henoch-Schonlein purpura. Endoscopy 2014; 46: 981-986.

18 Hung SP, Yang YH, Lin YT et al. Clinical manifestations and outcomes of Henoch-Schönlein purpura: comparison between adults and children. Pediatr Neonatol 2009; 50: 162-168.

19 Zhang Y, Huang X. Gastrointestinal involvement in Henoch-Schönlein purpura. Scand J Gastroenterol 2008; 43: 1038-1043.

20 Ilan Y, Naparstek Y. Schonlein-Henoch syndrome in adults and children. Semin Arthritis Rheum 1991; 21: 103-109.

21 Park CH, Han DS, Jeong JY et al. The optimal cut-off value of neutrophil-to-lymphocyte ratio for predicting prognosis in adult patients with Henoch-Schönlein purpura. PLoS ONE 2016; 11: e0153238.

22 Calvo-Río V, Loricera J, Ortiz-Sanjuán F et al. Revisiting clinical differences between hypersensitivity vasculitis and Henoch-Schönlein purpura in adults from a defined population. Clin Exp Rheumatol 2014; 32: S34S40.

23 Alalwani M, Billings SD, Gota CE. Clinical significance of immunoglobulin deposition in leukocytoclastic vasculitis: a 5-year retrospective study of 88 patients at cleveland clinic. Am J Dermatopathol 2014; 36: 723-729.

24 Hočevar A, Rotar Z, Jurčić V et al. IgA vasculitis in adults: the performance of the EULAR/PRINTO/PRES classification criteria in adults. Arthritis Res Ther 2015; 18: 58. 